\title{
Ripples and tails in the compact group of galaxies Hickson $54^{\star}$
}

\author{
L. Verdes-Montenegro, A. Del Olmo, J. I. Iglesias-Páramo, J. Perea, J. M. Vílchez, M. S. Yun, and W. K. Huchtmeier
}

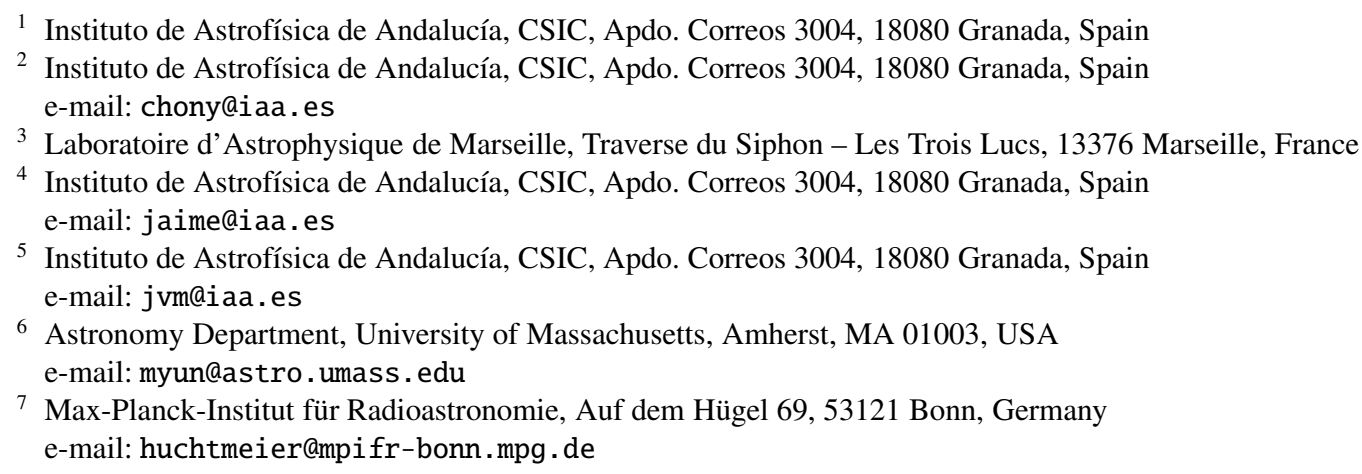

Received 16 May 2002 / Accepted 25 September 2002

\begin{abstract}
HCG 54 has been classified as a compact galaxy group by Hickson, but its nature is uncertain because of its unusual properties. We present here a study of HCG 54 based on deep optical images and spectroscopy as well as high spatial and spectral resolution HI observations obtained at the VLA. Both optical and radio data indicate clearly that HCG 54 is a product of a recent merger involving at least two galaxies. Our optical images have revealed a blue elongated structure associated with HCG 54a embedded in a rounder and redder stellar envelope. Several ripples or shells whose center is located near HCG 54a and $\mathrm{b}$ are also detected. These observed features are similar to those found in the numerical simulations of tidal interactions involving two equal mass disk galaxies. This system is embedded in an HI cloud $12 \mathrm{kpc}$ in diameter, and a $20 \mathrm{kpc} \mathrm{HI}$ tidal tail emerges from its western edge. Both the HI distribution and kinematics are consistent with a recent history of a deeply penetrating interaction or a merger. Based on the analysis of our new data we propose that HCG 54 is the remnant of a merger in an advanced stage, whose main body is what has been called HCG 54a, while HCG 54b marks the location of a strong starburst induced by the interaction, as evidenced by the Wolf Rayet stellar population that we detect. Optical spectra of HCG 54c and d show HII region features and are interpreted as tidal debris of the collision undergoing active star formation.
\end{abstract}

Key words. galaxies: interactions - galaxies: kinematics and dynamics - galaxies: evolution - galaxies: structure - radio lines: galaxies - galaxies: individual: HCG 54

\section{Introduction}

Hickson Compact Groups of galaxies are defined as aggregates of four or more galaxies with high galaxy densities similar to cluster cores (Hickson 1982) and low velocity dispersions $(<\sigma\rangle=200 \mathrm{~km} \mathrm{~s}^{-1}$, Hickson et al. 1992). The original catalog, composed of 100 groups, was produced without

Send offprint requests to: L. Verdes-Montenegro,

e-mail: lourdes@iaa.es

* Based on observations made with the VLA operated by the National Radio Astronomy Observatory (the National Radio Astronomy Observatory is a facility of the National Science Foundation operated under cooperative agreement by Associated Universities, Inc.) and on data taken using ALFOSC, which is owned by the Instituto de Astrofísica de Andalucía (IAA) and operated at the Nordic Optical Telescope under agreement between IAA and the NBIfA of the Astronomical Observatory of Copenhagen. kinematical information, and was later revised by Hickson et al. (1992) on the basis of redshifts obtained for most of the members of the groups. When chance projections of pairs and triplets with discordant galaxies were removed, the cata$\log$ was reduced to 69 groups. Still, reality of some of the proposed groups are questioned. For example, detailed investigations suggest that HCG 18 is probably a single knotty galaxy based on the HI and optical data (Williams \& Van Gorkom 1988; Plana et al. 2000).

HCG 54 was proposed by Hickson (1989) to be a small group composed of four dwarf galaxies, named a through d. The brightest galaxy has a "Sdm" morphological type while the rest are classified as "Im". Some authors have later claimed (e.g., Arkhipova et al. 1981) that HCG 54 is in fact a single galaxy composed of several bright HII regions. Vílchez \& Iglesias-Páramo (1998) found multiple emission knots in $\mathrm{H} \alpha$. 
HCG 54 occupies the lower end of the total luminosity, velocity dispersion and optical diameter distributions of the HCGs sample, with $\log \left(L_{B}\right)=9.27, \log \left(\sigma_{v}\right)=2.05$ and $R_{25}=4 \mathrm{kpc}$.

We were motivated to study HGC 54 in the context of our ongoing studies of HCGs (e.g. Verdes-Montenegro et al. 2001; Vílchez \& Iglesias-Páramo 1998) in order to confirm or discard its compact group nature based on optical images and spectra, together with HI VLA data. A large HI tidal tail, clear signature of a tidal interaction, as well as the presence of multiple optical ripples or shells, added new motivations to this study. In particular, the presence of ripples/shells and the apparent $\mathrm{Sdm}$ morphology pose an interesting theoretical problem, since the formation of these features has been generally attributed to a bright elliptical accreting a small companion (see e.g. Hernquist \& Quinn 1987). The observations are presented in Sect. 2, the results discussed in Sect. 3 and we present possible scenarios in Sect. 4. A distance of $19.6 \mathrm{Mpc}$ $\left(H_{0}=75 \mathrm{~km} \mathrm{~s}^{-1} \mathrm{Mpc}^{-1}\right)$ is assumed throughout this paper for HCG 54.

\section{Description of the observations}

\subsection{Optical imaging}

We have imaged HCG 54 in the Johnson $R$ band at the $2.5 \mathrm{~m}$ NOT telescope at el Roque de los Muchachos (La Palma) using the ALFOSC spectrograph during the nights of March 21st and 27 th and May 16th 2001. The main characteristics of the observations are given in Table 1 . The detector was a Loral/Lesser (CCD\#7) $2048 \times 2048$, with a spatial scale of $0.189^{\prime \prime} /$ pixel, which gives a field of about 6! $5 \times 6$ ' 5 . In order to cover a larger field (16." $6 \times 16$." 7) tracing the direction of the HI tail (see Sect. 3.3), a total of 10 shifted exposures were taken during the $1 \mathrm{st}$ run for $600 \mathrm{~s}$ each. The seeing was varying between 1." 1 and 1." 4 during the observations. Ten bias exposures were taken through this run and were used to construct a median bias which was subtracted from each image. Pixel-to-pixel variations were evaluated with a median normalized twilight flatfield. Then a bidimensional fourth order Legendre polynomial was fitted using IMSURFIT task in IRAF. Images were divided by this flat field. After correction of instrumental effects, the atmospheric extinction was determined and corrected from observations of selected fields from Landolt list of standard stars (PG0918 and PG1047). The rms errors of the standard stars in the final calibration are smaller than $0.07 \mathrm{mag}$. Finally all individual images were combined in a mosaic by using the IRAF SQIID package created by K. M. Merrit at NOAO, and using SQMOS, XYGET and NIRCOMBINE routines, together with a mask built for each frame that prevented the mean of the frame edges, as well as bad CCD lines. The resulting image is shown in Fig. 1, where three previously unclassified galaxies A1126+2051, A1127+2054 and A1127+2057 are also identified (see Sects. 3.1 and 3.2).

In the second run, a deeper red band exposure was obtained for the central part in order to better define the faintest optical structures. The red image is a composition of six $1200 \mathrm{~s}$ images with the Sloan-Gunn $r^{\prime}$ filter. A blue image was also obtained in order to be able to derive colour indices of the
Table 1. Photometric observations.

\begin{tabular}{llcl}
\hline \hline Image & Date & $T_{\mathrm{ex}}$ & Filter \\
\hline Mosaic & $21 / 03 / 2001$ & 6000 & $R$-Johnson \\
Center & $27 / 03 / 2001$ & 7200 & $r^{\prime}$ Gunn \\
Center & $16 / 05 / 2001$ & 4800 & $B$-Johnson \\
\hline
\end{tabular}

different components of the group. The Johnson $B$ filter image taken in the 3rd run is a composition of four $1200 \mathrm{~s}$ frames. Slight dithering was applied between successive frames for both filters, in order to avoid cosmic rays and bad pixels. All frames ( $R, r^{\prime}$ and $B$ band images) were finally combined in a search for the best definition of the weakest structures (Fig. 2). None of the nights of these two last runs were photometric, so no calibration was obtained for the $r^{\prime}$ and $B$ band images. Instead we adopted the one proposed by Hickson et al. (1989) for the $(B-R)$ color map (Fig. 3), using the integrated $B-R$ color of HCG $54 \mathrm{~b}$ as a reference.

\subsection{Optical spectroscopy}

The spectra were also obtained with ALFOSC at the NOT $2.5 \mathrm{~m}$ telescope, with the same detector used for the images and using Grism\#4, 7 and 8, during April 2000 and March 2001. Table 2 summarizes the long-slit spectra taken for this study. The format is as follows: Col. 1 spectrum identification, 2 direction through which the spectrum was taken, 3 date of observation, 4 Grism used, 5 spectral dispersion, 6 number of exposures, 7 duration of each exposure in seconds, 8 spectral range, 9 position angle of the slit in degrees measured from $\mathrm{N}$ to $\mathrm{E}$, and 10 width of the slit. The slit widths were chosen to match approximately the seeing of the nights. The direction of the slits listed in Table 2 as well as the different zones from which the spectra have been extracted along the slits are marked in Fig. 4. Observations with Grism\#4 were performed in order to derive general spectral characteristics and kinematics of the galaxies, and to determine redshifts for possible satellite galaxies in the field. Grisms\#7 and \#8 were used to characterize the kinematics with a higher spectral resolution than for Grism\#4. Observations with Grism\#7 covered with good spectral resolution the range including [OII]3727 and [SII]6717/6731. In the observations performed with Grism\#8 the [NII], $\mathrm{H} \alpha$ and [SII] lines were detected and used to characterize the kinematics.

The spectra were reduced according to the usual methods, including subtraction of a mean bias calculated for each night, division by a median flat-field obtained for each configuration, as well as wavelength calibration based on HeNe calibration lamp spectra obtained after and before each exposure. The rms of the bidimensional calibration was $0.4 \AA$ for Grism\#4 and $0.1 \AA$ for Grism\#7 and Grism\#8. A mean sky was obtained from object-free sections of each spectrum. In all cases several exposures were taken in order to increase the signal to noise ratio and to remove cosmic rays. The derived physical parameters described in Sect. 3.2 are given in Tables 3 and 4.

In order to obtain redshifts and velocity dispersions we used the cross-correlation technique derived by Tonry \& Davis (1979). The templates used for the spectra showing 


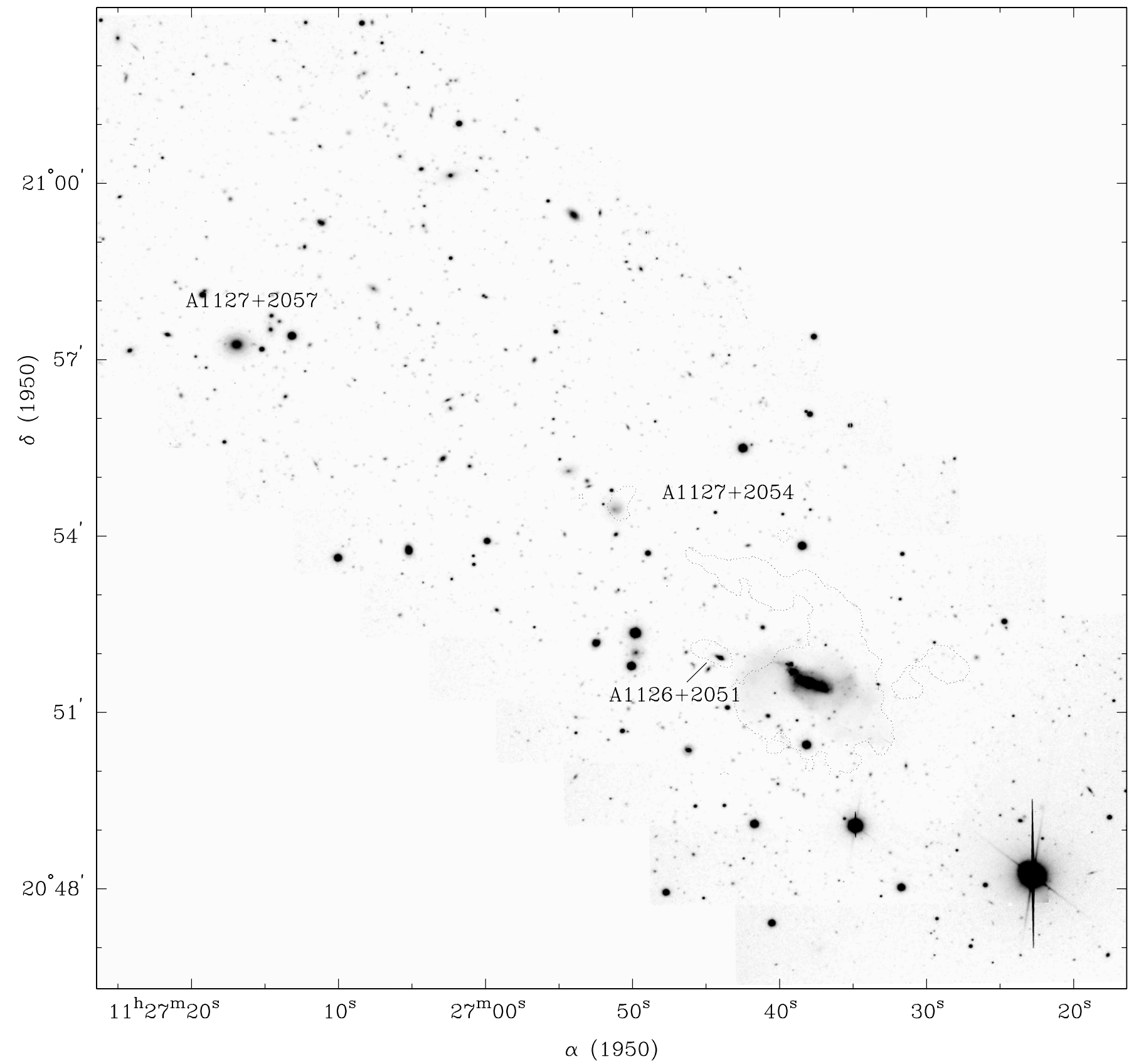

Fig. 1. Full field of the $R$-Johnson band optical image of HCG 54 obtained as explained in the text (Sect. 2.1). The dotted lines delineate the HI integrated emission at a level of $0.5 \times 10^{20}$ atoms $\mathrm{cm}^{-2}$. We have labeled the three dwarf galaxies discussed in the text (Sect. 3.2).

emission lines were: the brightest spatial section of HCG $54 \mathrm{a}$ and HCG 54c for the Grism\#8 and Grism\#7 spectra respectively, together with a synthetic spectrum built from the rest frame wavelengths of the emission lines. In the case of the absorption line spectra, where our goal was to determine the redshift of A1126+2051 and A1127+205, we used as template the spectrum of the radial velocity standard giant star HD186176 obtained with the same configuration. In order to improve the signal to noise ratio of the derived curves a binning of 2 pixels $\left(0 .{ }^{\prime \prime} 38\right)$ has been applied to the spectra sp5 and sp6 in the spatial direction and of 3 pixels ( $\left.0 .{ }^{\prime \prime} 57\right)$ to sp1 (Fig. 4). The radial velocity data in the direction joining HCG 54a and b are shown in Fig. 5a, in the HCG $54 \mathrm{c}-\mathrm{d}$ direction in Fig. 5b together with the section of sp1 corresponding to HCG $54 \mathrm{~d}$, and in the
HCG 54d - A1126+2051 in Fig. 5c. The spectra for HCG 54a and $b$ taken with Grism\#4 are shown in Fig. 6 and 7 respectively. Spectrophotometric standard stars were observed in order to get a proper calibration of the line fluxes in sp3 and sp4 (Fig. 4). However, since the nights of April 27 and 28 were non-photometric, only the relative fluxes of the emission lines are reliable. The rest of spectra were taken under photometric conditions.

\subsection{VLA HI observations}

We have mapped HCG 54 with the VLA in the C array in August 1997. The synthesized beam is $20^{\prime \prime} \times 16^{\prime \prime}(1.9 \times$ $1.5 \mathrm{kpc}$ at a distance of $19.6 \mathrm{Mpc}$ ) after natural weighting of the 


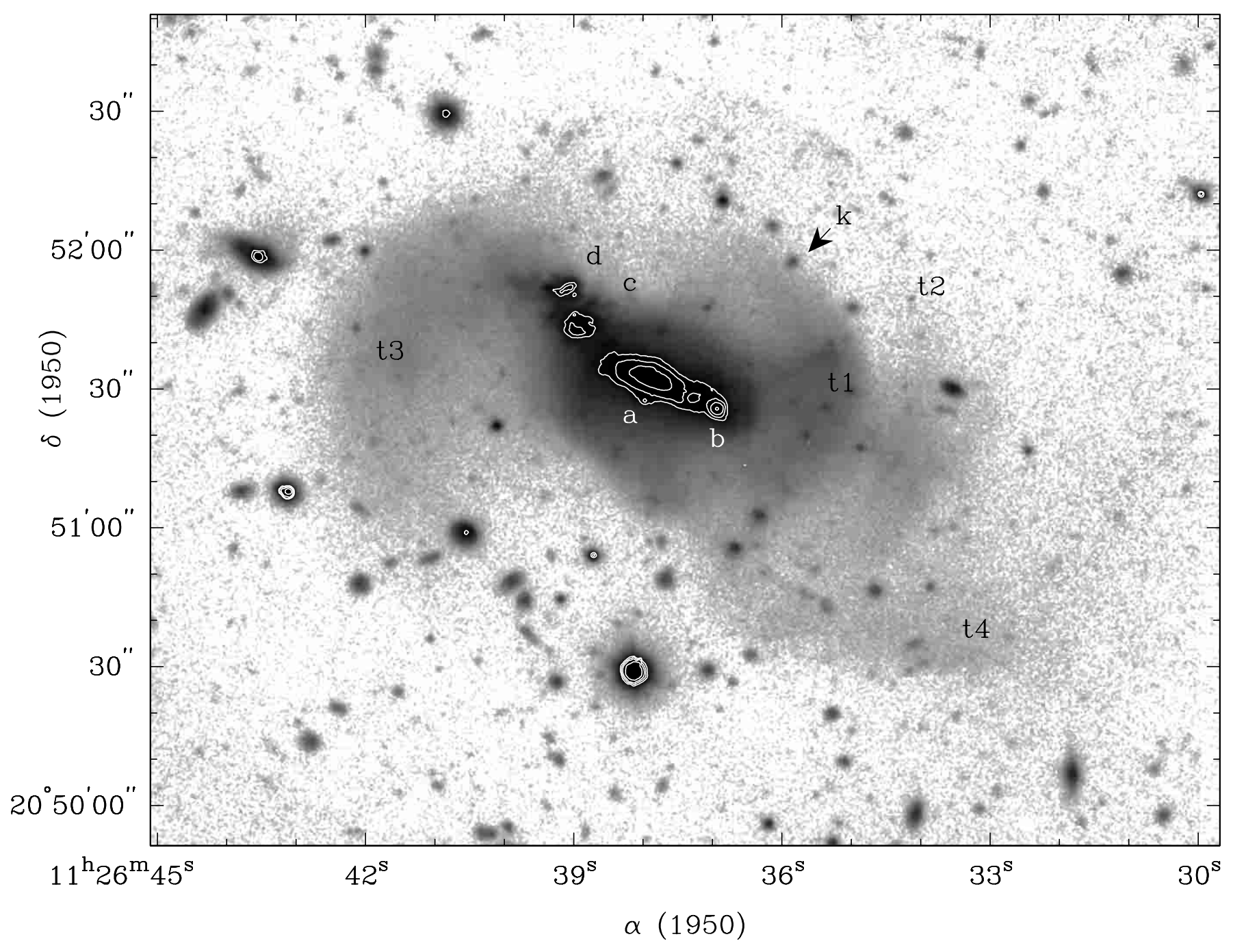

Fig. 2. Combined frame of $B, R$ and $r^{\prime}$ bands in order to better recover the faintest emission. Contours are shown for the central part that appears saturated in our greyscale. The objects identified by Hickson et al. (1989) as the members of HCG 54 are indicated with letters a to d. We also mark the tidal optical features described in Sect. $3.1 t 1$ to $t 4$, and an example of the unresolved knots (k) that we detect close to HCG 54 .

Table 2. Spectroscopic observations.

\begin{tabular}{llcccccccc}
\hline \hline Name & Direction & Date & Grism & $R(\AA / \mathrm{px})$ & $N$ & $T_{\text {exp }}(\mathrm{s})$ & Wav.-range $(\AA)$ & PA (deg) & Slit width $(")$ \\
\hline sp1 & HCG 54d-A1126+2051 & $21 / 03 / 01$ & GR4 & 3.0 & 3 & 1800 & $3018-9006$ & 86 & 1.2 \\
sp2 & A1127+2057 & $21 / 03 / 01$ & GR4 & 3.0 & 2 & 1200 & $3018-9006$ & 115 & 1.2 \\
sp3 & HCG 54a-b & $27 / 03 / 01$ & GR4 & 3.0 & 3 & 1200 & $3018-9006$ & 65 & 1.0 \\
sp4 & HCG 54c-d & $28 / 03 / 01$ & GR4 & 3.0 & 3 & 1200 & $3018-9006$ & 20 & 1.0 \\
sp5 & HCG 54a-b & $27 / 04 / 00$ & GR8 & 1.2 & 3 & 1800 & $5816-8326$ & 65 & 1.2 \\
sp6 & HCG 54c-d & 21/03/01 & GR7 & 1.5 & 2 & 1200 & $3815-6815$ & 15 & 1.2 \\
\hline
\end{tabular}

data. A velocity range between $1085 \mathrm{~km} \mathrm{~s}^{-1}$ and $1730 \mathrm{~km} \mathrm{~s}^{-1}$ was covered with a velocity resolution of $10.4 \mathrm{~km} \mathrm{~s}^{-1}$. The rms noise is $0.33 \mathrm{mJy} /$ beam corresponding to a column density of $1.2 \times 10^{19}$ at $\mathrm{cm}^{-2}$. Assuming an HI linewidth of $30 \mathrm{~km} \mathrm{~s}^{-1}$, the achieved $\mathrm{HI}$ mass detection limit is about $10^{6} M_{\odot}{ }^{1}$.

\footnotetext{
${ }^{1}$ Computed as $M(\mathrm{HI})=2.36 \times 10^{5} D^{2} S \Delta V$, where $D$ is the distance in $\mathrm{Mpc}$ and $S \Delta V$ is the velocity integrated HI flux in Jy $\mathrm{km} \mathrm{s}^{-1}$.
}

We have detected emission above $3 \sigma$ in the velocity range 1334.2 to $1490.2 \mathrm{~km} \mathrm{~s}^{-1}$. The integrated emission is presented in Fig. 8a superposed on the $R$-band image, and the velocity field is presented in Fig. 8b. The velocity channel maps are shown in Fig. 9. The total spectrum has been obtained by integrating the emission in the individual channel maps (Fig. 10, solid line). The total HI line flux detected, $6.23 \mathrm{Jy} \mathrm{km} \mathrm{s}^{-1}$, 


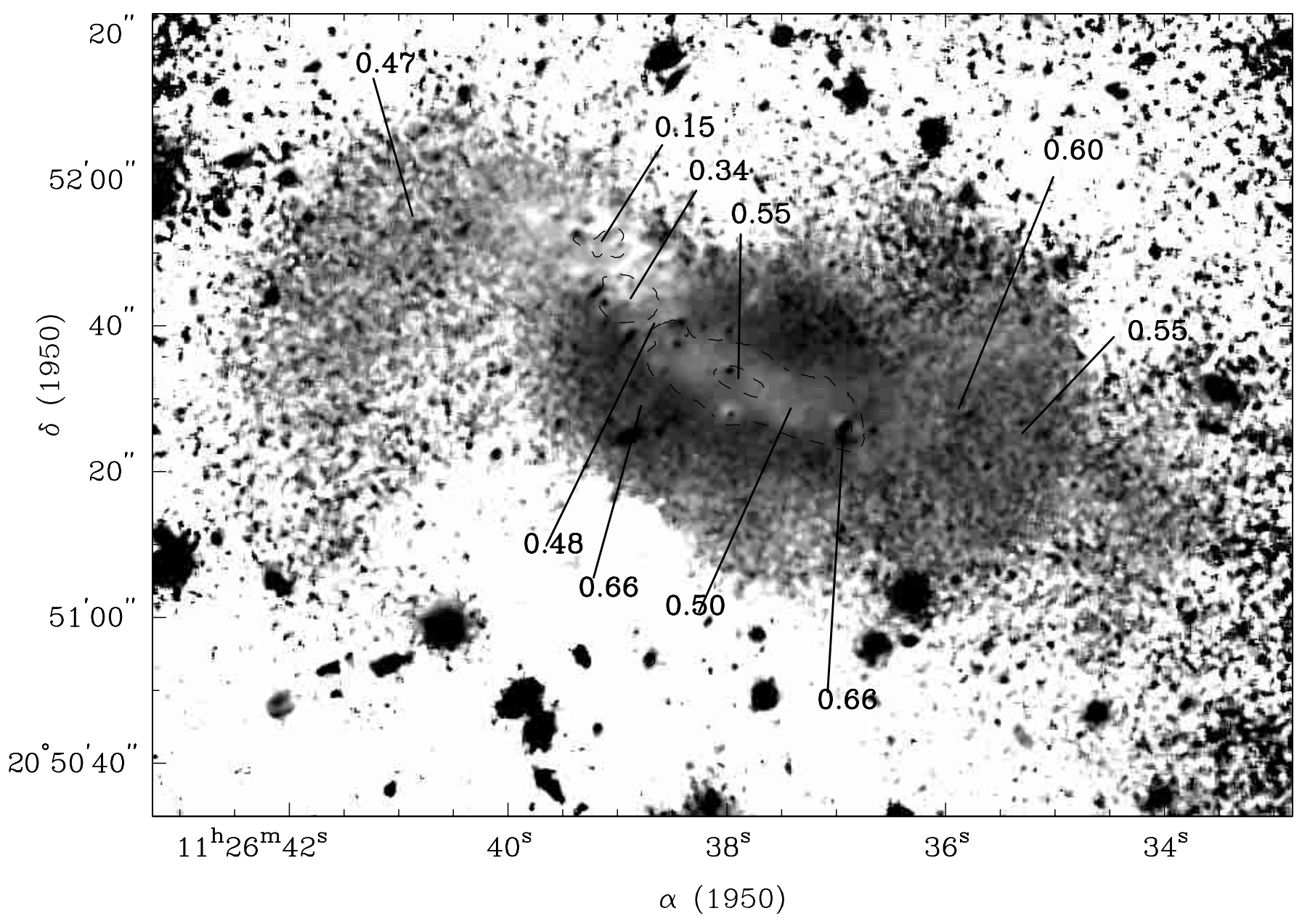

Fig. 3. $B-R$ map of HCG 54. Averaged colors of different regions are indicated by arrows, and the displayed range goes from 0 (white) to 0.8 (black). We have superimposed two $R$ band isophotes as a reference.

is in good agreement with the single dish measurement by Huchtmeier (1997; Fig. 10, dotted line). The total HI mass derived is $5.6 \times 10^{8} M_{\odot}$.

\section{Results}

\subsection{Optical images}

The brightest central part of HCG 54 (Fig. 2) shows a dominant knot (HCG 54a) embedded in an elongated feature visible in the blue and red bands at a PA of $65^{\circ}$. It is more extended to the SW, where a very compact bright knot is located (HCG 54b) which is even more prominent in $\mathrm{H} \alpha$ (see Vílchez \& IglesiasPáramo 1998). The elongated area defined by HCG 54a and b is connected to the NE with the weaker but still prominent knots HCG 54c and d.

Large variations in the $B-R$ color index are apparent throughout the whole system (from 0.15 in HCG 54d to 0.70 around HCG 54a, Fig. 3). The elongated structure centered in HCG 54a has a blue color (0.5), likely a signature of a recent star forming episode, and is embedded in a rounder region that shows the reddest colours in HCG $54(B-R=0.7)$.

The $B-R$ color index for HCG $54 \mathrm{~b}$ is surprisingly red for a strong star formation burst like the one hosted by this galaxy. However, we argue that this is due to the contamination by the $\mathrm{H} \beta$, [OIII] and $\mathrm{H} \alpha$ emission lines. After computing the equivalent widths of these lines $(E W(\mathrm{H} \beta)=$ $155 \AA, E W([\mathrm{OIII}] 4959)=246 \AA, E W([\mathrm{OIII}] 5007)=728 \AA$, $E W(\mathrm{H} \alpha+[\mathrm{NII}])=798 \AA$ ), we estimated that the corrected $B-R$ color should be 0.46 mag bluer than the one measured directly from the color map. Thus, we would obtain $B-R=0.20$, which is more consistent with a very young stellar population.

Three shell-like structures are found (noted as $t 1$ to $t 3$ in Fig. 2), centered roughly on HCG 54a and b. A fourth optical feature is located to the SW and marked as $t 4$. We detect all of these features in a $R$-band surface brightness range of 24 to $27 \mathrm{mag} /\left({ }^{\prime \prime}\right)^{2}$. The outer shells ( $t 2$ and $t 3$ ) although redder than the internal knots, have blue colours $(\sim 0.5)$ typical of irregular galaxies (see Fukugita et al. 1995). The inner shell $(t 1)$ is slightly redder (0.60), probably due to contamination from the central parts of HCG 54. Numerous unresolved knots are detected everywhere in the area, such as the one marked in Fig. 2 with a " $k$ ". No stellar counterpart to the HI long tail is detected down to $27 \mathrm{mag} /\left({ }^{\prime \prime}\right)^{2}$ in $R$.

We found three faint galaxies in our large $R$-band frame (Fig. 1), that are named A1126+2051, A1127+2057 and A1127+2054. The first two are background galaxies (Sect. 3.2) while the last one is at the velocity of the system according to 


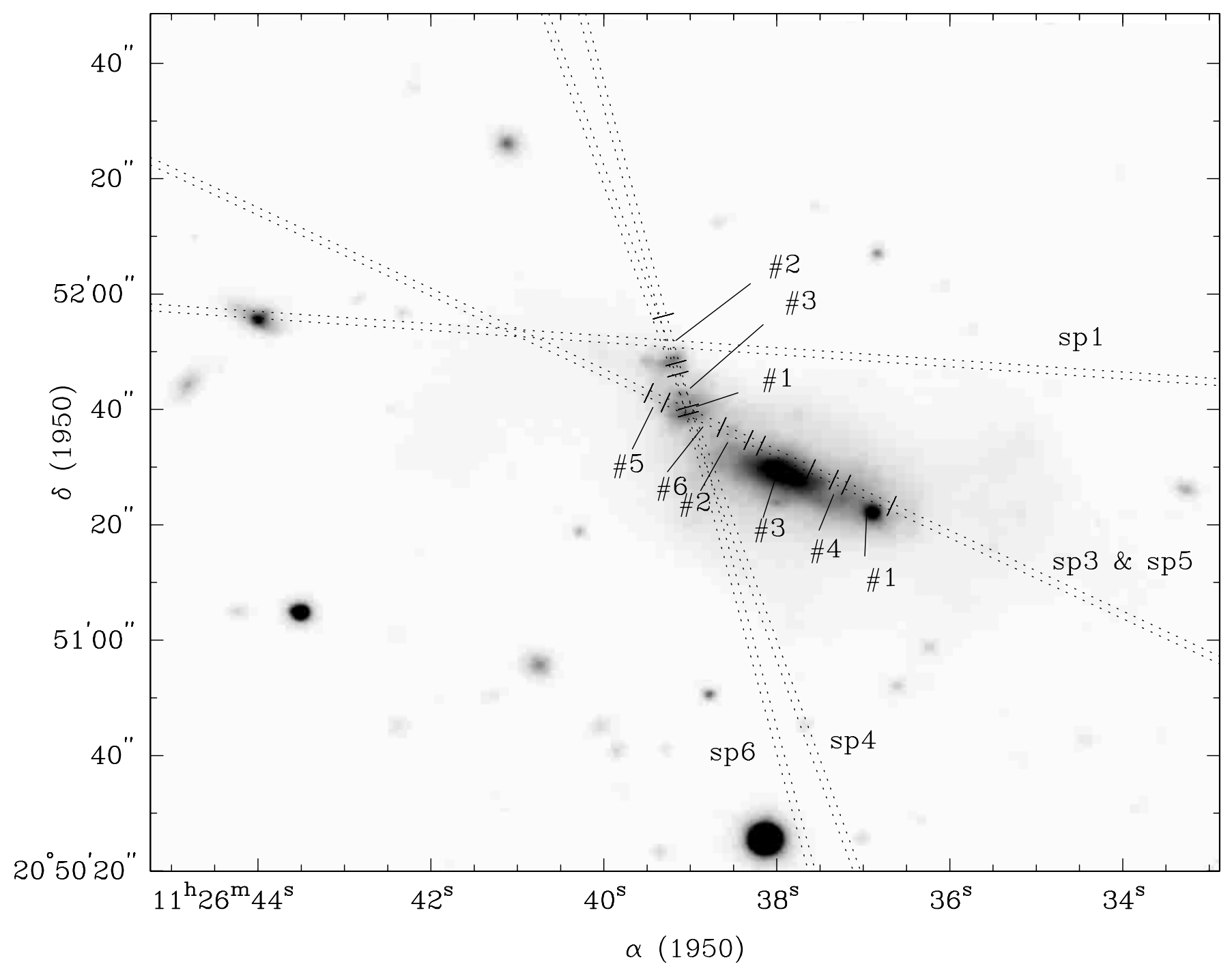

Fig. 4. $R$ band image of HCG 54 showing the direction of the slits used for the spectroscopical observations in the main body of HCG 54 (see Sect. 2.2), and the sections indicated in Tables 3 and 4.

the HI data (Sect. 3.3). The optical image of this galaxy shown in Fig. 11 reveals that the inner and the outer isophotes are off-centered. When measured at an $R$ isophote of $26 \mathrm{mag} /\left({ }^{\prime \prime}\right)^{2}$ the optical size is $2.6 \mathrm{kpc}$ and the total magnitude in $R$ is $M_{R}=-14.2 \mathrm{mag}$. The surface brightness derived as a function of radius is exponential, consistent with that of a disk system (see Fig. 11b).

\subsection{Description of the spectra}

From the Grism\#4 spectra sp1 (Fig. 4) and sp2 (Table 2) we have determined that A1126+2051 and A1127+2057 are not at the group redshift. A1126+2051 shows absorption lines of CaII, G band, $\mathrm{Mg}$ I and $\mathrm{Na}$ lines and has a redshift of $z=0.15$ (see also Sect. 3.3). A1127+2057 also shows an absorption spectrum typical of early type galaxies and gas at a redshift of $z=0.052$.

We have studied the kinematics of the central parts of the group with the spectra obtained with Grism\#7 and \#8. The spectrum along the HCG 54a-b direction (sp5, Fig. 4) shows emission lines along the 249 spatial sections ( $\left.47^{\prime \prime}\right)$, and the obtained velocity curve is plotted in Fig. 5a, where the zero position corresponds to the center of HCG 54b. We mark in the figure the continuum extent of HCG 54a and mark with an arrow the position of the emission line maxima. The spatial sections closer to HCG 54a (5" diameter) traces a distorted rotation curve with an amplitude of $45 \mathrm{~km} \mathrm{~s}^{-1}$. At both sides of the center of HCG 54a we find discontinuities in the velocity that correspond in the $R$ band to distorted isophotes. The sections in the direction of HCG $54 \mathrm{c}$ ( $R 1$ in Fig. 5a) have three well differentiated knots with emission lines, whose left end is the external part of HCG 54c. The strongest knot presents a velocity gradient of $50 \mathrm{~km} \mathrm{~s}^{-1}$ in $4^{\prime \prime}$ that corresponds to the contact area between HCG 54a and HCG 54c $(R 3)$. The velocities in the direction of HCG 54b show irregular changes of up to $70 \mathrm{~km} \mathrm{~s}^{-1}$, as is the case of $R 2$ (Fig. 5a) that appears to be an HII region detached from the general trend of the velocity curve. The area of HCG 54b has a peculiar kinematics (Fig. 5a) that could be 


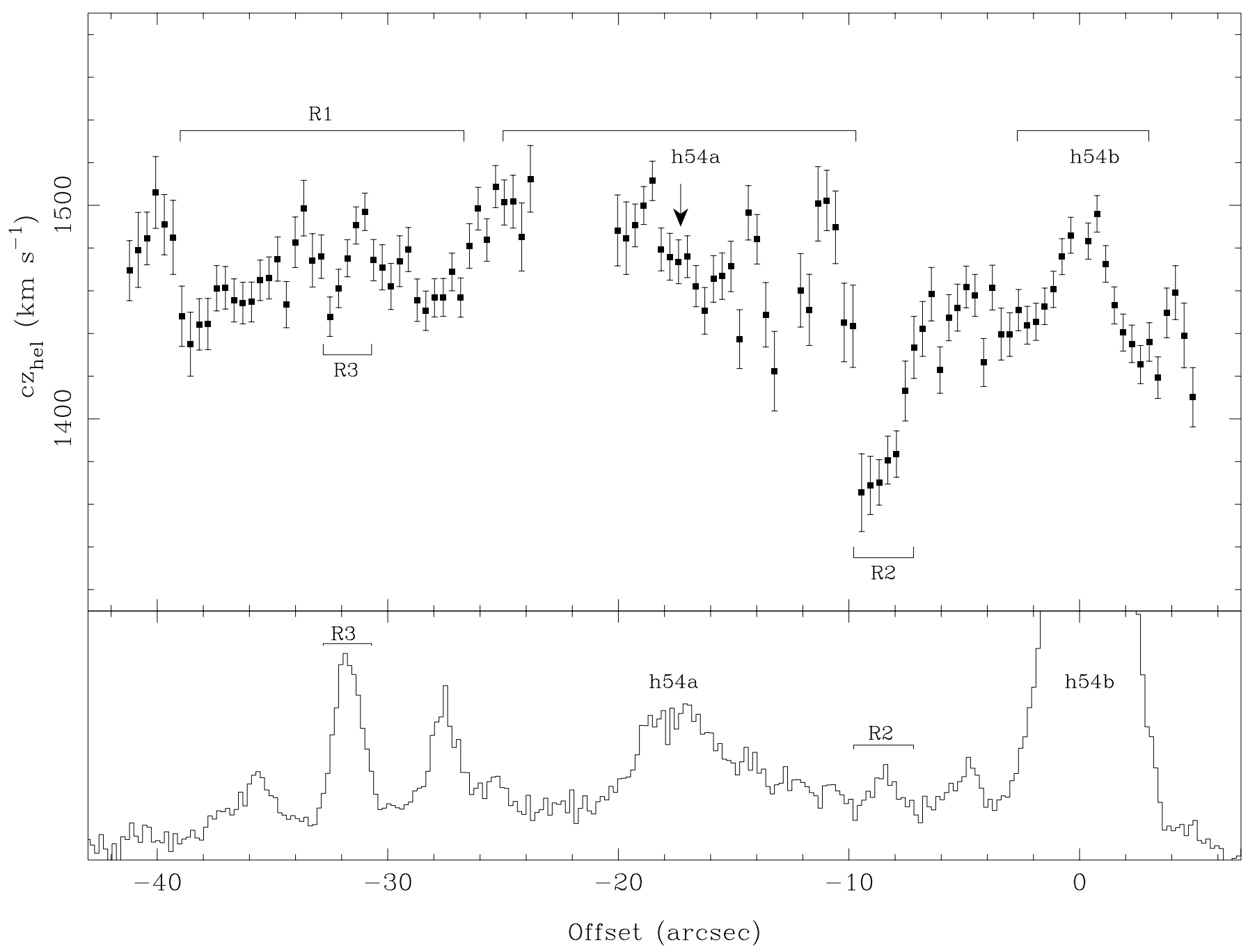

Fig. 5. a) Up: spectrum in the direction joining HCG 54a and b (sp3), where the position is given in offsets with respect to the peak of emission of HCG 54b. The indicated regions are described in Sect. 3.2. Bottom: $R$ band profile along the slit.

consistent with infalling gas, in the case that the background emission is hidden by extinction so that we are only observing the motions of the foreground component.

In Fig. 5b we show the velocities in the slit through HCG 54c-d (sp6, Fig. 4), and the regions corresponding to HCG $54 \mathrm{c}$ and d in the $R$ image are also marked. These regions have a weak continuum, and none of them show a rotation curve. HCG 54d has irregular motions, while HCG 54c shows a nearly constant velocity following a U-shape with an amplitude $30 \mathrm{~km} \mathrm{~s}^{-1}$. We do not detect continuum emission toward the third emitting region in the direction of HCG 54a (Fig. 5b), while the velocities increase continuously until it reaches the values characteristic of HCG 54a. Finally we find a region with a decoupled velocity $(R 4)$ that is located between HCG $54 \mathrm{c}$ and $\mathrm{d}$

In the lower resolution spectrum crossing HCG 54d (sp1, Fig. 4) we detect a velocity gradient of only $\sim 70 \mathrm{~km} \mathrm{~s}^{-1}$ within $7^{\prime \prime}$. In Fig. 5c we show the sections where we have detected emission, where the center corresponds to the continuum peak of HCG 54d along the slit. The presence of several knots in our $R$ band image along the slit direction suggests that the continuity of the observed velocity gradient might be due to smearing of individual components.

We have extracted individual spectra for 9 zones from the data taken with Grism\#4 (sp3 and sp4, Fig. 4). Six of them are along the slit position joining HCG $54 \mathrm{a}-\mathrm{b}$ and three along the HCG 54c-d direction (Fig. 4). The spectrum of HCG 54a (sp3, Fig. 4) shows strong Balmer absorption lines as well as lines of CaII, Gband, and possibly MgI, while $\mathrm{Mg}_{2}$ is barely detected. The spectrum shows a blue continuum, characteristic of a post-starburst population (Fig. 6). The spectrum corresponding to knot $\mathrm{b}$ is remarkable (Fig. 7), with a high excitation and the presence of WR features at $\sim 4650 \AA$ over a flat continuum, indicative of a young and strong burst of star formation. Figure $7 \mathrm{a}$ shows the full spectrum of HCG 54b, while Fig. 7b shows a detail of the spectrum around the Wolf-Rayet (WR) feature. The temperature sensitive [OIII] line at $\lambda 4363 \AA$ was measured in this spectrum, giving a $t([\mathrm{OIII}])$ temperature of $10485 \mathrm{~K}$, implying an oxygen abundance of $12+\operatorname{LogO} / \mathrm{H}=8.26$ (see Table 3 ). According to 


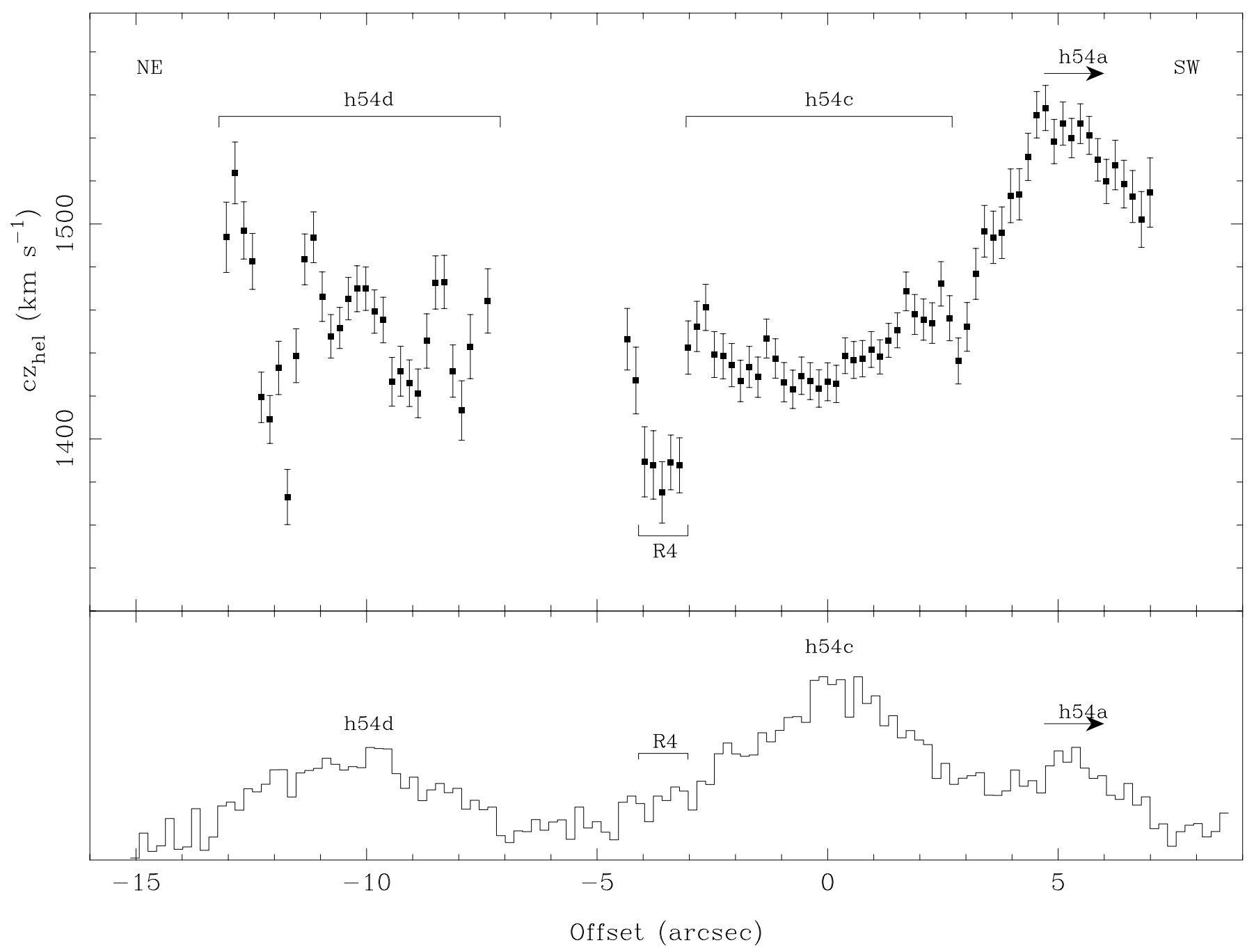

Fig. 5. b) Up: spectrum in the direction joining HCG 54c and d (sp4), where the position is given in offsets with respect to the peak of emission of HCG 54c. Bottom: $R$ band profile along the slit.

Schaerer \& Vacca (1998), the measured equivalent width of the WR $\lambda 4650 \AA$ feature in knot b, $E W(\mathrm{WR})=9 \pm 0.9 \AA$, implies an age of the burst between 3 and 4 Myr.

For the zones showing emission line spectra (sp3, sp4, Fig. 4) we present in Tables 3 and 4 their fluxes relative to $\mathrm{H} \beta$ as well as the derived physical conditions of the ionized gas. Reddening corrected line fluxes relative to $\mathrm{H} \beta$ are presented for the 9 individual spectra extracted along slit positions sp3 and sp4 (Table 2, Fig. 4). For each zone given in Tables 3 and 4 the following ionization structure parameters have been derived in order to perform the abundance analysis (as detailed below): $R_{23}$, denotes the abundance parameter after Pagel et al. (1980) which is defined as $[I(3727)+$ $I(5007)+I(4959)] / I(\mathrm{H} \beta), P$ denotes the abundance parameter defined by Pilyugin (2000), as quoted in Tables 3 and 4, $P=[1.3 \times I(5007) / I(\mathrm{H} \beta)] / R_{23}$. The excitation, defined as $1.3 \times I(5007) / I(3727)$, and the nitrogen to oxygen abundance indicator, $1.3 \times I(6584) / I(3727)$, are also quoted in Table 2 . Note the small range of variation of $R_{23}$ in contrast with the large variations shown by the excitation along the slit. The electron temperatures $t[\mathrm{OIII}]$ and $t[\mathrm{OII}]$, corresponding to the ionization zones of [OIII] and [OII] respectively, have been derived for region \#1 and listed in Table 3 . For this region the ionic and total abundances of oxygen, $\mathrm{O}^{2+} / \mathrm{H}^{+}, \mathrm{O}^{+} / \mathrm{H}^{+}$and $\mathrm{O} / \mathrm{H}$ respectively, as well as the abundance ratio of neon to oxygen, $\mathrm{Ne}^{2+} / \mathrm{O}^{2+}$, have been calculated and are presented in the table. For the rest of the studied regions, only the total abundance of oxygen has been estimated (see Tables 3 and 4).

Given that the temperature sensitive line [OIII]lambda 4363A was measured only for knot b, we have to rely on the empirical calibration in order to derive their abundances. We have used the calibration of the oxygen abundance versus $R_{23}$ (cf. Pagel et al. 1980) as parameterized by McGaugh in Kobulnicky et al. (1999), and following the $P$-method (Pilyugin 2000 ), in order to provide an estimation of $12+\log \mathrm{O} / \mathrm{H}$. The $[\mathrm{NII}] /[\mathrm{OII}]$ line ratio, when observed, was used to discriminate between the lower and upper branch, though often the 6584 line was severely blended with $\mathrm{H} \alpha$ and was not measured. For those zones with $R_{23} \geq 0.9$ we have assumed an average abundance of $12+\log \mathrm{O} / \mathrm{H}=8.2$ as indicated by the calibration. All the zones selected are consistent with $12+\log \mathrm{O} / \mathrm{H}=8.2$, within 


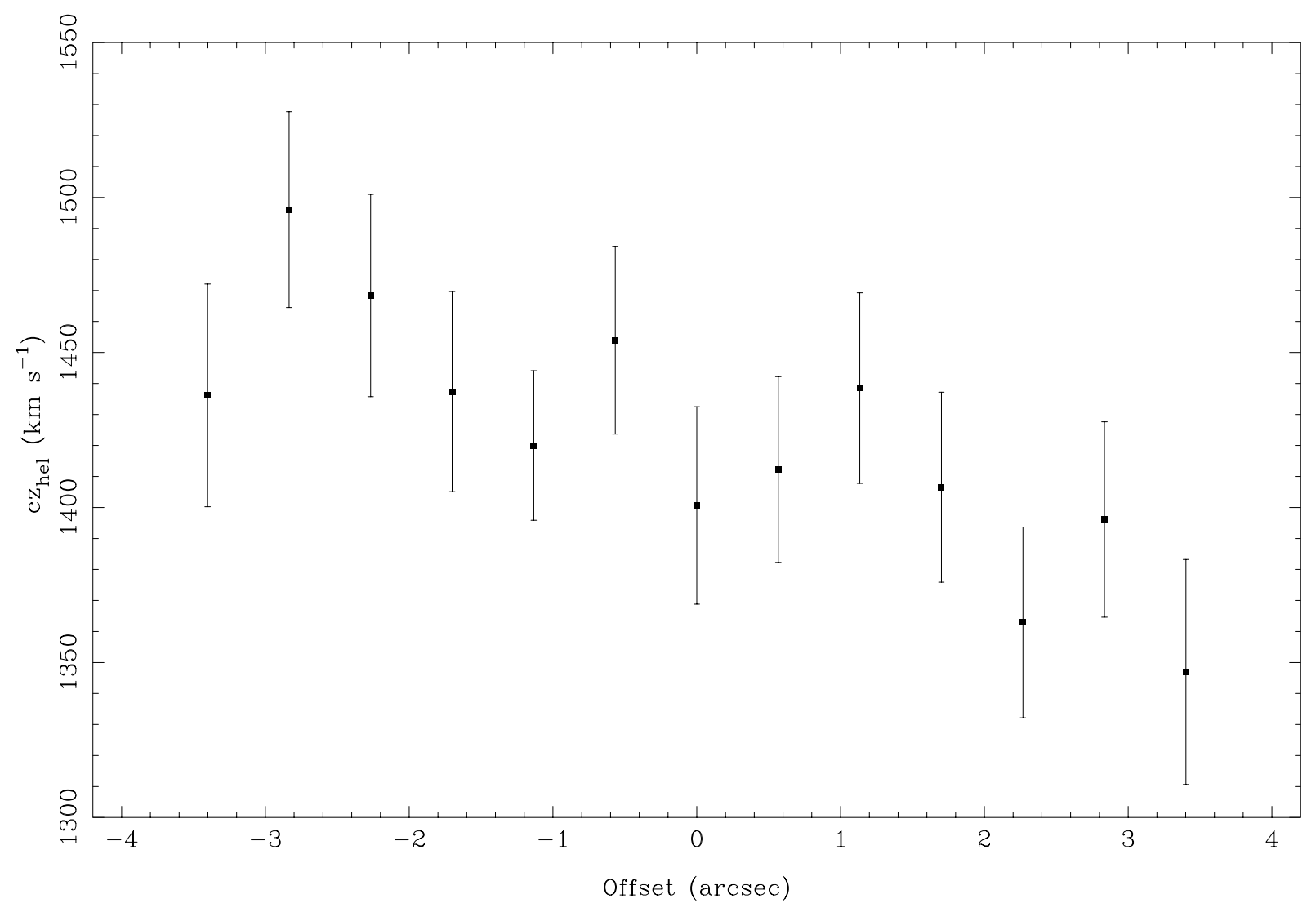

Fig. 5. c) Spectrum in the direction joining HCG 54d and A1126+2051 (sp1, Fig. 4), for the sections where emission was detected. Positions are given in offsets with respect to the continuum peak of HCG $54 \mathrm{~d}$ along the slit.

the errors of the empirical calibration. This abundance is typical of galaxies like the Large Magellanic Cloud and the outer disks of late type spirals.

\subsection{Neutral hydrogen}

The integrated emission of neutral hydrogen (Fig. 8a) shows a NE-SW distribution with extensions to the SE and SW, and a long tail with a projected size of $20 \mathrm{kpc}$ to the NE. The velocity field is quite perturbed, but still shows a velocity gradient similar to a rotating disk with a twisted major axis (Fig. 8b). This reflects itself also in the asymmetry of the HI line integrated profile (Fig. 10).

The situation is more complex when the channel maps (Fig. 9) are examined. The sizes of the optical knots are small compared with the VLA synthesized beam, and tracing the HI kinematics with respect to the optical features is difficult. Nevertheless these channel maps reveal the details that are lost in the integrated emission image shown in Fig. 8a. Except for the large HI tail to the northeast, most of the HI emission arises within the faint optical extent of HCG 54. Bright HI features directly associated with the bright optical ridge of emission are seen in the channel maps with velocity range between 1365 $1490 \mathrm{~km} \mathrm{~s}^{-1}$. The overall velocity field is along the length of the bright optical ridge in a manner consistent with that of rotation, but clear evidence for a velocity gradient in the perpendicular direction is also present, increasing in velocity from NW to SE. Since stars and gas inside the tidal radius are generally unaffected by a tidal interaction, the observed kinematic disturbance suggests an involvement of a deeply penetrating interaction or a merger.

Most of the high surface brightness HI features associated with the fainter outer optical envelope occur on the west side of the optical galaxy, closely associated with the optical tidal features $t 1, t 2$, and $t 4$ (see Fig. 2). Both the $Y$-shaped HI morphology in Fig. 8a and the shifting of the focus of the iso-velocity contours to the west of the optical peaks in Fig. 8b are direct results of the large amount of $\mathrm{HI}$ associated with these tidal features. This one-sided appearance may indicate that only one of the progenitor systems was HI-rich if HCG 54 is mainly a product of a merger involving two late type galaxies - i.e. the progenitor responsible for the tidal feature $t 3$ had relatively little HI associated with its stellar disk. The HI extension to the southwest, closely associated with the optical feature $t 4$, occurs mainly in the velocity ranges of 1334 to $1428 \mathrm{~km} \mathrm{~s}^{-1}$ and contains $1.4 \times 10^{8} M_{\odot}$ of HI. The long HI tail associated with either $t 1$ and/or $t 2$ occurs in the velocity range of 1376 to $1459 \mathrm{~km} \mathrm{~s}^{-1}$ and contains about $10^{8} M_{\odot}$ of HI. Combined together, these two outer $\mathrm{HI}$ features account for more than $40 \%$ of the total HI detected in this system.

Several HI clumps that are detached from the main body of $\mathrm{HI}$ are also seen at $3-5 \sigma$ levels in these channel maps, and they indicate a more extensive debris field associated with this system. A velocity coherent string of HI clumps forming 


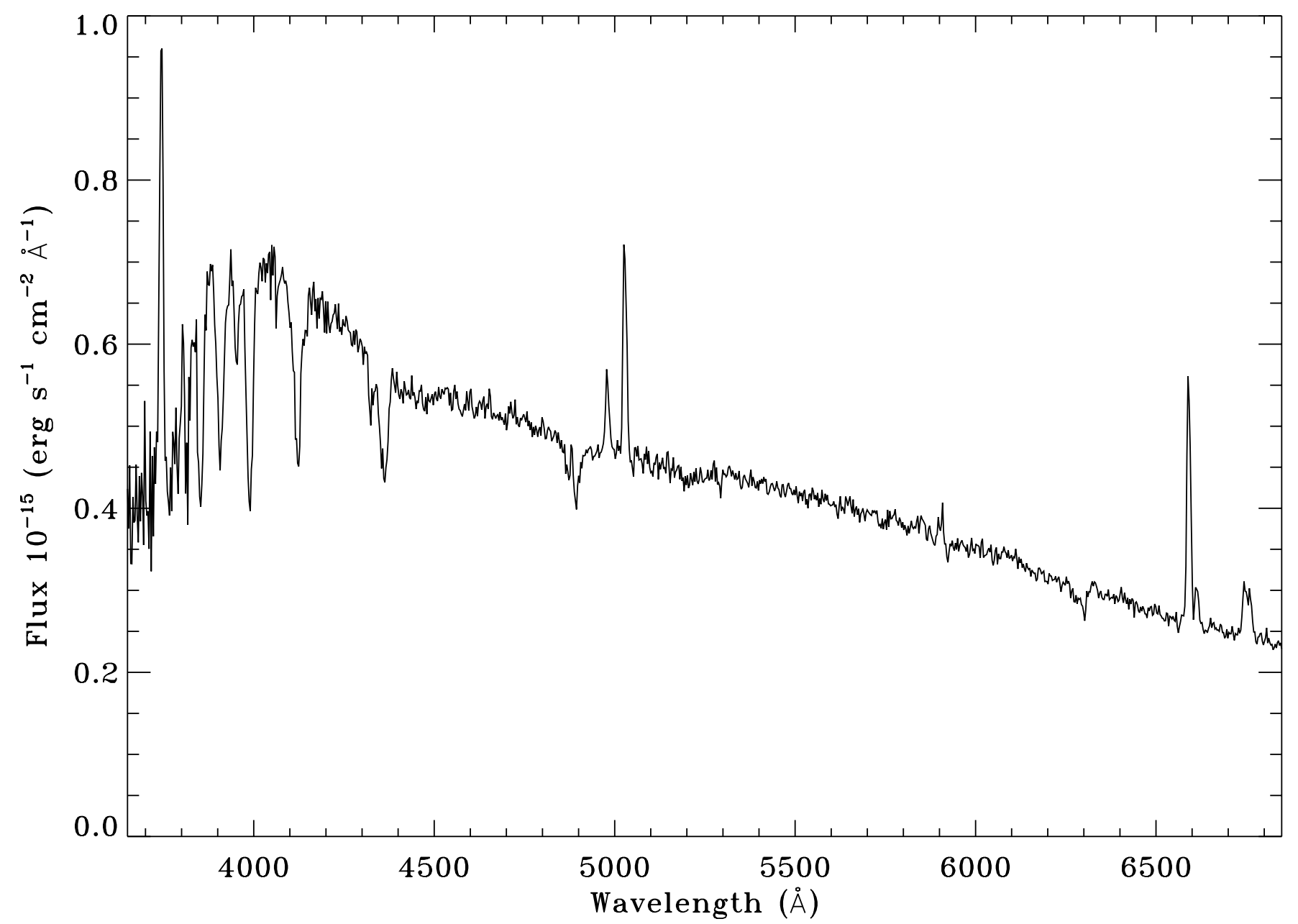

Fig. 6. Full spectrum of HCG 54a.

a nearly complete loop or a ring is seen in the channel maps within the velocity range of 1438 to $1469 \mathrm{~km} \mathrm{~s}^{-1}$, largely to the northeast. Some of these features make up part of the $20 \mathrm{kpc}$ long HI tail to the north, but their appearance in the channel maps, particularly at $1438 \mathrm{~km} \mathrm{~s}^{-1}$ and $1448 \mathrm{~km} \mathrm{~s}^{-1}$, suggest a more ring-like morphology, similar to those seen in collisional ring galaxies (e.g. Higdon 1996). The stream of HI clumps extending to the west of the main body seen in Fig. 8a form a second distinct, velocity coherent structure appearing at velocities of 1386-1407 $\mathrm{km} \mathrm{s}^{-1}$, and these channel maps suggest its origin being more to the south of the main body rather than a linear east-west structure. The total HI mass associated with this feature is about $3 \times 10^{7} M_{\odot}$.

The overall velocity field of the bulk of HI delineates an elongated structure with an axis ratio that would correspond, if intrinsically circular, with an approximate inclination of $50^{\circ}$ and a position angle of $70^{\circ}$, similar to the one traced by the direction of HCG $54 \mathrm{a}-\mathrm{b}\left(\mathrm{PA}=65^{\circ}\right)$. The amplitude derived from the velocity field $\left(140 \mathrm{~km} \mathrm{~s}^{-1}\right)$ and deprojected according to this inclination gives an overall velocity gradient of $\sim 183 \mathrm{~km} \mathrm{~s}^{-1}$. If this is interpreted as a Keplerian rotation, we estimate a dynamical mass for the system of $M_{\mathrm{tot}} \sim 10^{10} M_{\odot}$.

A dwarf companion galaxy A1127+2054 is found at a projected distance of $27 \mathrm{kpc}$ northeast of HCG 54a (see Sect. 3.1,
Figs. 1 and 11). Its location also corresponds to a distance of $8 \mathrm{kpc}$ from the tip of the $20 \mathrm{kpc}$ long HI tail, in the same projected direction. The HI channel maps show associated emission in 3 channels covering velocities of $1386-1407 \mathrm{~km} \mathrm{~s}^{-1}$. A close examination suggests a central depression in the integrated emission (see Fig. 11c). The velocity field is consistent with a slowly rotating disk with a velocity amplitude of $30 \mathrm{~km} \mathrm{~s}^{-1}$ (Fig. 11d). The HI extent at $3 \times 10^{19}$ at $\mathrm{cm}^{-2}$ $(3 \sigma$ level $)$ is $45^{\prime \prime} \times 25^{\prime \prime}(4.3 \mathrm{kpc} \times 2.4 \mathrm{kpc})$ and contains $1.9 \times 10^{7} M_{\odot}$ of HI. The atomic component seems to be perturbed since it is not well centered on the optical component.

In the superposition of the integrated $\mathrm{HI}$ emission over the deep optical image shown in Fig. 8a, the HI isolated peak located about $2^{\prime}$ east of HCG 54a appears to have an optical counterpart in a bright, compact galaxy. From the optical spectrum we obtain, however, that this is a chance superposition as this galaxy A1126+2051 is found to be a background object with $z=0.15$ (see Sect. 3.2). At the position of this galaxy we find HI emission in two channel maps, with no signature of systematic motions. Since the gas is located close to the center of the group, it is possibly part of the tidal debris. This is supported when a sharpening of the optical image of A1126+2051 is performed, suggesting the existence of two objects, a symmetric disk structure whose peak is located in the slit, and then 


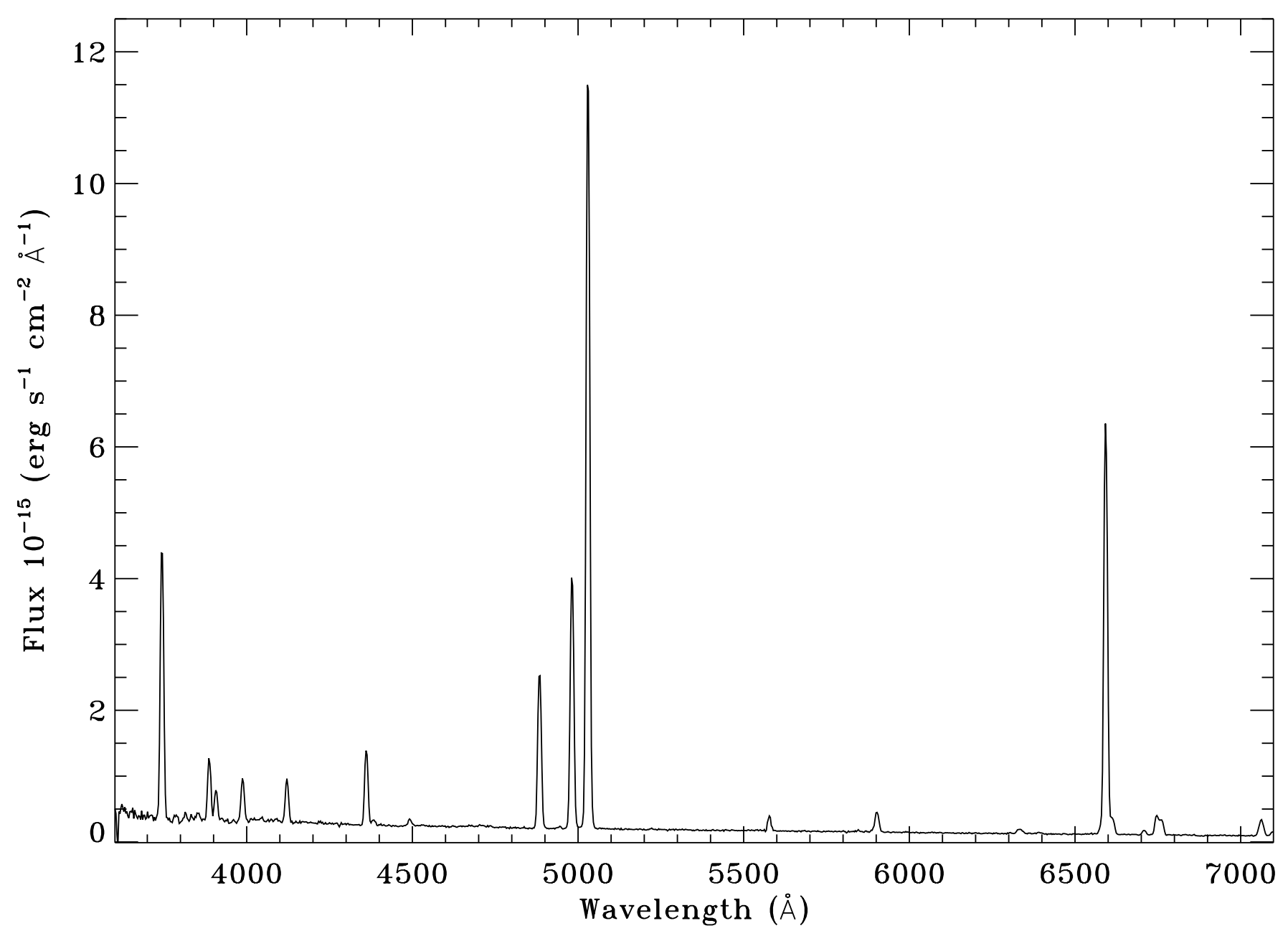

Fig. 7. a) Full spectrum of HCG 54b.

corresponds probably to the background galaxy, plus a blob that might be a stellar counterpart of the HI emission.

\subsection{Radio continuum emission}

Using the data from the line-free channels, a $1.4 \mathrm{GHz}$ continuum image was constructed. No significant continuum emission associated with the optical galaxy is detected at the $3 \sigma$ limit of about $0.5 \mathrm{mJy}^{\text {beam }}{ }^{-1}$. The New VLA Sky Survey image also gives a comparable upper limit of about $1.5 \mathrm{mJy}$ for about a 3 times larger beam. HCG 54 was detected by IRAS in the $12 \mu \mathrm{m}, 60 \mu \mathrm{m}$, and $100 \mu \mathrm{m}$ bands with flux densities of $0.24,0.50$, and $0.84 \mathrm{Jy}$, respectively, with the derived FIR luminosity of $L_{\mathrm{FIR}}=3 \times 10^{8} L_{\odot}$ (Verdes-Montenegro et al. 1998). The upper limit of $1.5 \mathrm{mJy}$ for $1.4 \mathrm{GHz}$ radio continuum makes this object slightly underluminous in the radio with a lower limit on the $q$-value of about 2.7 (see Yun et al. 2001). The enhanced IR luminosity resulting from the relatively high dust temperature, inferred from the FIR $\left(S_{60} / S_{100}=0.60\right)$ and mid-IR $\left(S_{25} / S_{60}=0.48\right)$ color, offers a natural explanation for the relatively weak radio continuum. These warm infrared colors are also consistent with the evidence for significant WR activity detected in the optical spectra (see Sect. 2.2).

\section{Discussion}

We have identified clear signs of tidal interactions and possibly a merger toward HCG 54. The morphology and kinematics of the atomic gas, a sensible tracer of interactions, is strongly perturbed, including a long $(20 \mathrm{kpc}) \mathrm{HI}$ tidal tail, plus two more HI extensions that emerge from the main body. The stellar component also shows features that are characteristic of interactions or mergers, in particular several shells around the bright central area. We propose here that the formation of the HI tail and the optical ripples involved interactions and a merger of at least two galaxies.

\subsection{The isolated environment of HCG 54: only one (dwarf) companion}

The proximity of HCG 54 to our Local Universe $(v=$ $1470 \mathrm{~km} \mathrm{~s}^{-1}$ ) makes the study of its large scale environment difficult. We find in the NASA Extragalactic Database $(\mathrm{NED})^{2}$ that all galaxies with measured redshifts in a square

${ }^{2}$ This research has made use of the NASA/IPAC Extragalactic Database (NED) which is operated by the Jet Propulsion Laboratory, California Institute of Technology, under contract with the National Aeronautics and Space Administration. 


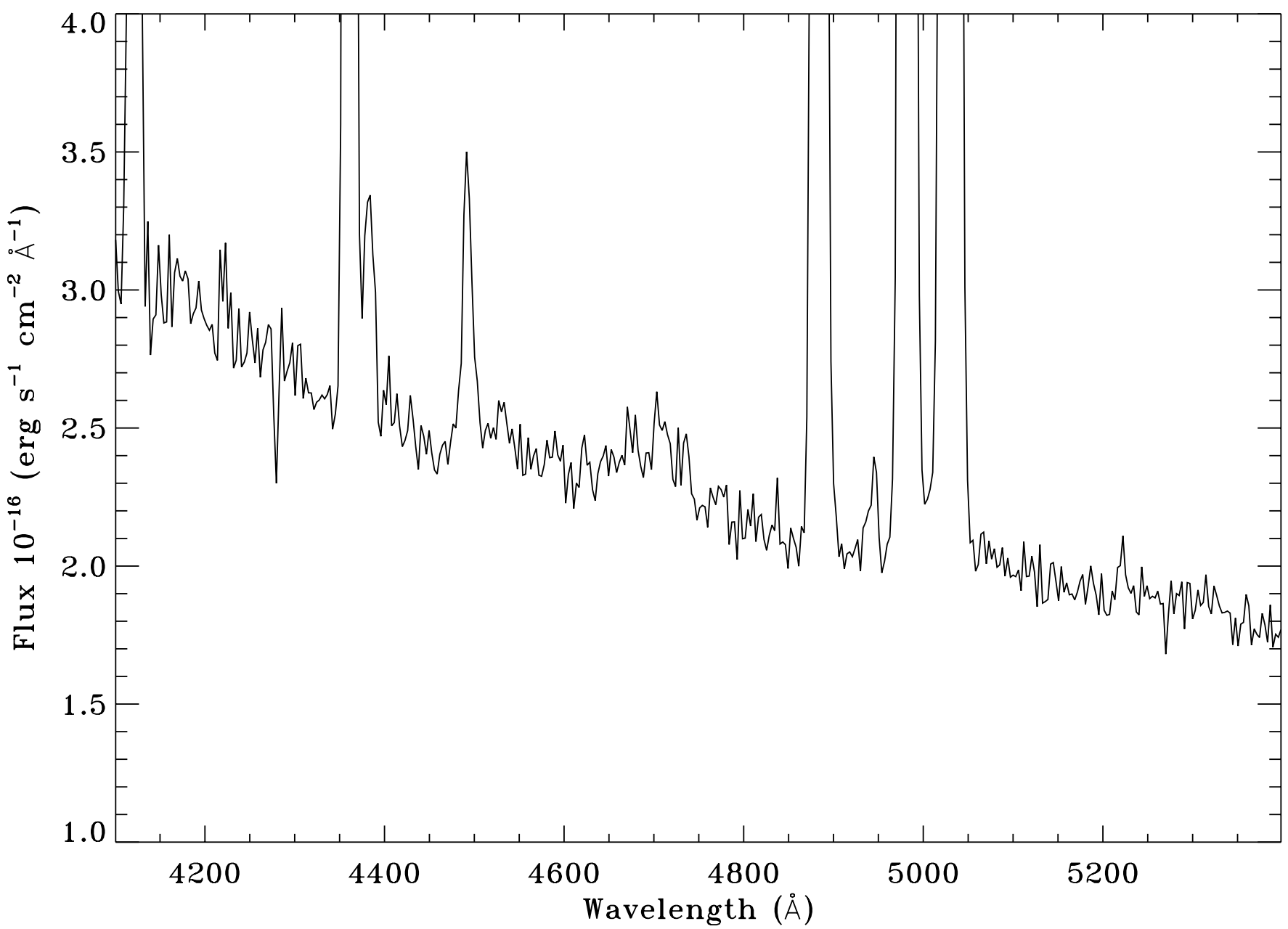

Fig. 7. b) Selected range of the same spectrum shown in a).

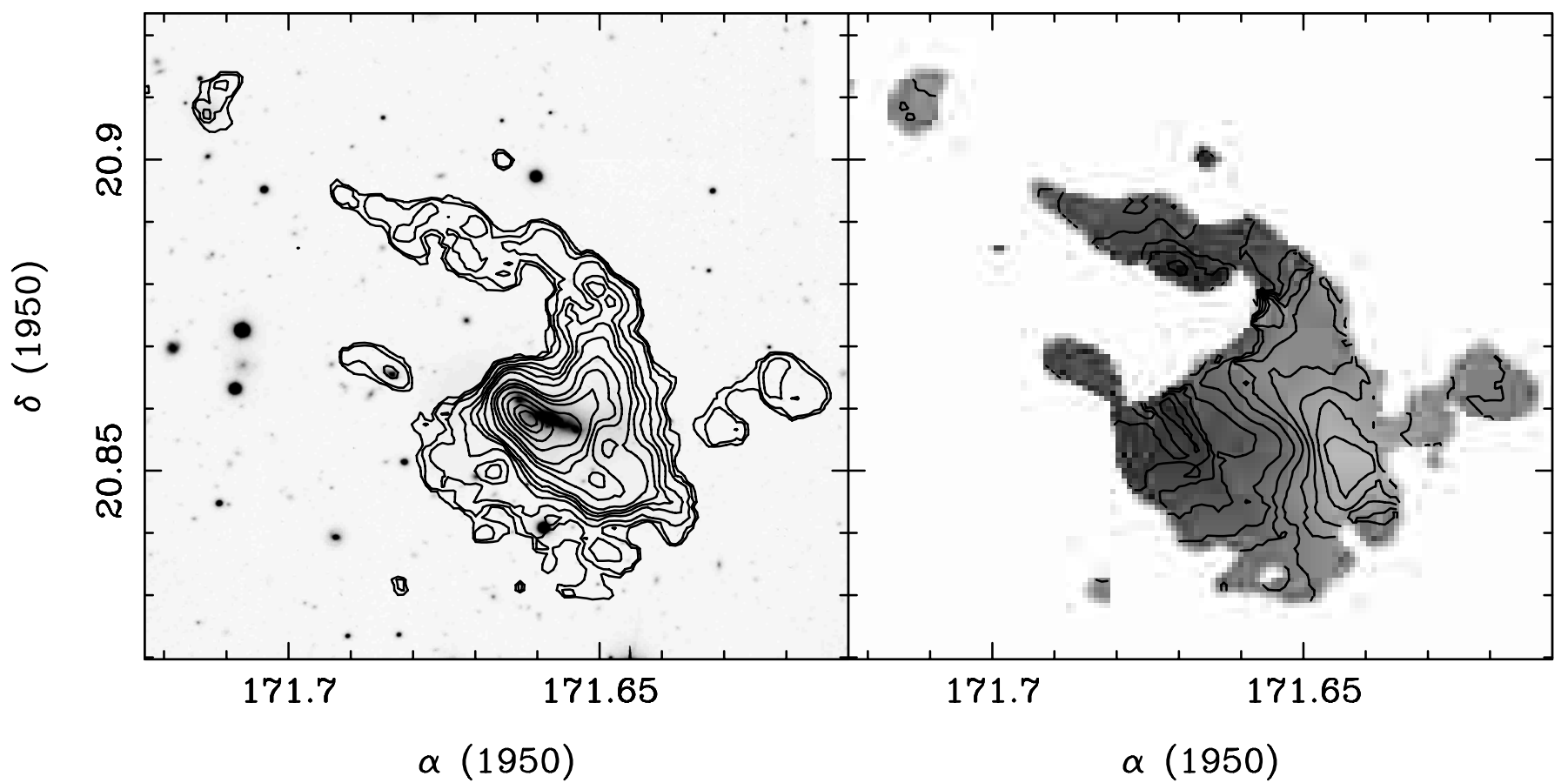

Fig. 8. a) Left: HI column density contours $0.5,0.7,1.2,2.0,2.7,3.4,4.4,6.7,8.4,10.1,13.5,16.9,20.2$ to $22.6 \times 10^{20}$ atoms $\mathrm{cm}^{-2}$ overlapped on the R image of HCG 54. The atomic emission has been integrated in the velocity range 1334-1490 $\mathrm{km} \mathrm{s}^{-1}$. The synthesized beam is $20^{\prime \prime} \times 16^{\prime \prime}$. b) Right: map of the first-order moment of the HI radial velocity field. The scale goes as in the wedge where the numbers indicate heliocentric velocities in $\mathrm{km} \mathrm{s}^{-1}$. The contours go from 1340 to $1490 \mathrm{~km} \mathrm{~s}^{-1}$ with a step of $10 \mathrm{~km} \mathrm{~s}^{-1}$. The beam size is $20^{\prime \prime} \times 16^{\prime \prime}$. 


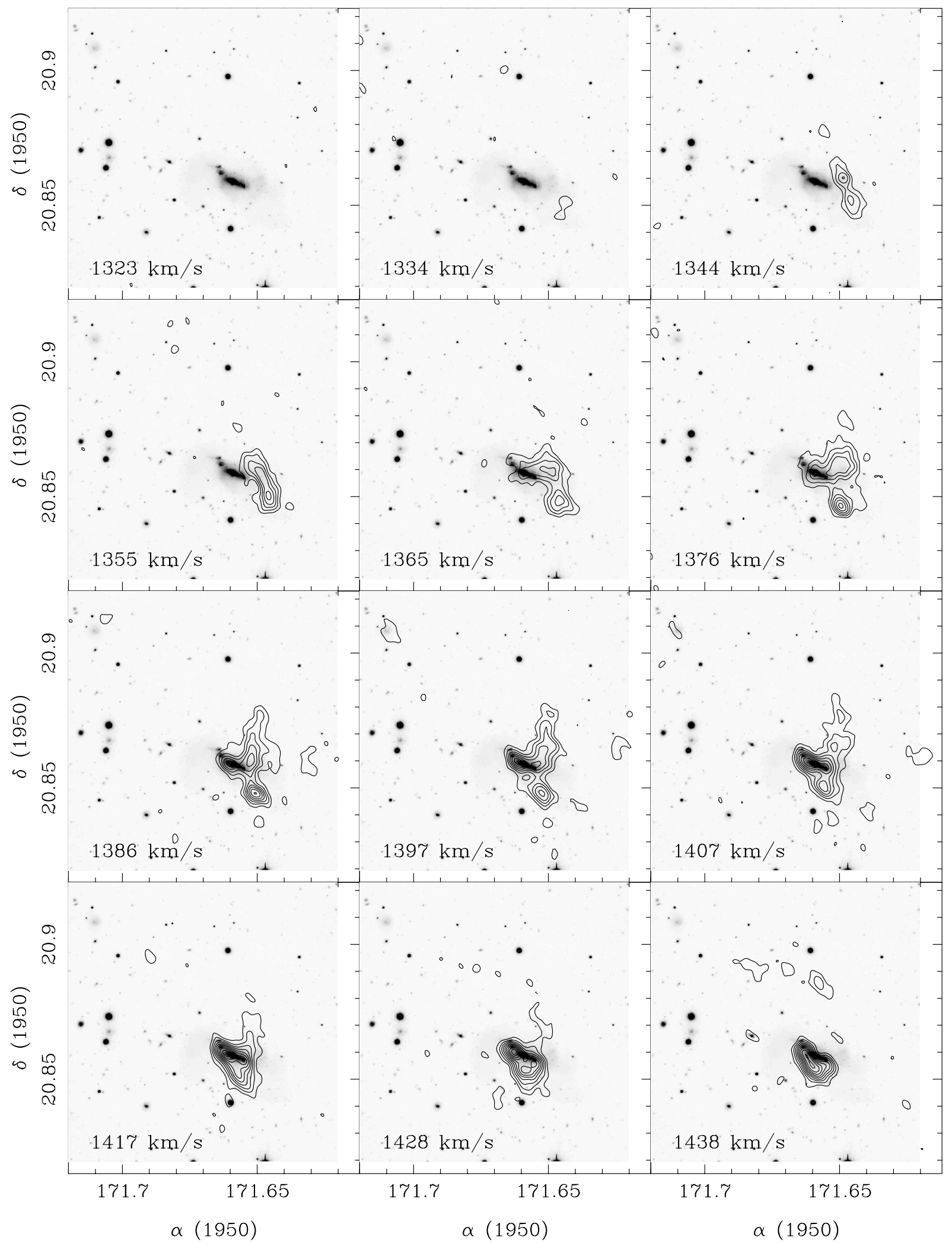

Fig. 9. Channel maps of the $21 \mathrm{~cm}$ line radiation superimposed on the $R$ image of the group. Heliocentric velocities are indicated in each panel. Contours correspond to $2.4,4.0,5.6,7.3,8.9,10.5,12.1,13.7,15.3 \mathrm{~K}$, and the rms noise of the maps is $1.2 \mathrm{~K}$. The synthesized beam $\left(15\right.$." $^{\prime} \times$ 14 ." 5 ) is plotted in the upper left panel.

of $1 \mathrm{Mpc}$ radius centered in HCG 54 have velocities larger than $4000 \mathrm{~km} \mathrm{~s}^{-1}$. Within a distance of $500 \mathrm{kpc}$ none of the galaxies without redshift determination have a similar size or magnitude as the HCG 54 members. In fact, the closest and brightest galaxy to HCG 54, A1127+2057, is a background galaxy (see Sect. 3). Similarly, another optically selected 


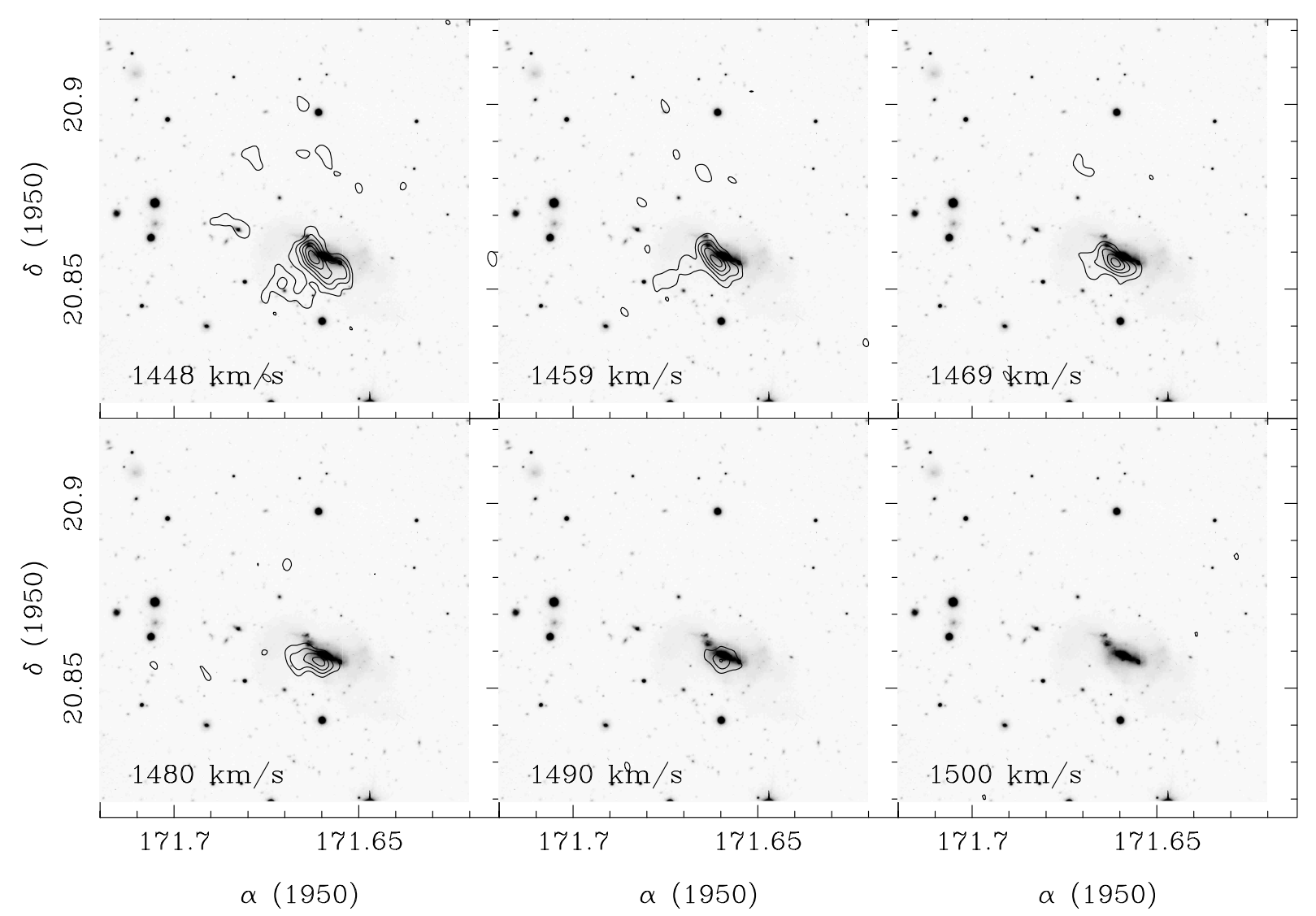

Fig. 9. continued.

potential neighbor A1126+2051 is also shown to be a background galaxy.

The VLA primary beam $\left(30^{\prime}\right)$ covers a diameter of $170 \mathrm{kpc}$ at the distance of HCG 54, and we found only one object $(\mathrm{A} 1127+2054$, see Sect. 3$)$ in this area at the group redshift in $\mathrm{HI}$ emission, within the observed range of $645 \mathrm{~km} \mathrm{~s}^{-1}$. The dwarf disk galaxy A1127+2054 is the only identified companion in the environment of HCG 54 and is located at $20 \mathrm{kpc}$ from its center.

\subsection{Origin of the stellar ripples/shells}

Shells are usually thought to form in unequal mass interactions involving a bright elliptical accreting a small companion (see e.g. Quinn 1984; Hernquist \& Quinn 1987). The work by Schweizer \& Seitzer (1988) showed that a smooth triaxial potential, or even a disk, can also give rise to the formation of shells and ripples in the stellar component of an interacting smaller companion. It does not seem to be the case in HCG 54, where we do not detect a large bulge component nor any other massive system. The two galaxies should have been most probably late-type systems, judging from the poststarburst nature of the spectrum shown by HCG 54a (without clearly detected features typical of early type systems) which could be invoked as the possible nucleus of the system. Furthermore, the low chemical abundance derived from the ionized gas, $12+\log (\mathrm{O} / \mathrm{H}) \sim 8.3$, is more typical of irregular galaxies. Few examples of shells around low mass late type galaxies are found in the literature, as NGC 7673 (Homeier \& Gallagher 1999). If we relax the low mass criteria there is also NGC 3310 (see Mulder \& van Driel, 1996) which is a peculiar Sbc. In both cases a tidal interaction origin has been attributed to the shells. In general, little observational evidence exists for ripples in disk-disk interactions, (see e.g. Homeier \& Gallagher 1999; Charmandaris \& Appleton 1996; Kemp \& Meaburn 1993 or Schweizer \& Seitzer 1988). Probably for this reason few models are found focused on reproducing HCG 54-like systems. In this sense, the simulations presented by Hernquist \& Spergel (1992) seem to provide with a more suitable model to describe the system. The model represents a merger of two equal disks galaxies in a close collision from a parabolic orbit. At the final stages the remnant has a central bar-like structure embedded in a disk like envelope, together with several shells at different radii. This resembles quite well the optical structure of HCG 54, centered on HCG 54a, with an associated bar-like bluer structure, whose colours are suggestive of star formation, in the middle of a red disk-like component and surrounded by 3 shells. The shells are centered somewhere close to HCG 54a and $b$, but the data do not allow a determination of the precise location of their focus (Sect. 3.1). The outer shells ( $t 1 \& t 3$, see Fig. 2) have bluer colors, consistent with an origin in the late type progenitor disk. The inner one $(t 2)$ is slightly redder, and this might be due to contamination by the population of the disk-like component associated with HCG 54a. Therefore the morphology of HCG 54 can be well explained by the collision of two similar mass disk systems. The model does not allow 
Table 3. Line fluxes relative to $\mathrm{H} \beta$ and abundances ${ }^{1}$ across slit position through HCG 54a-b.

\begin{tabular}{|c|c|c|c|c|c|c|c|}
\hline ION & $\lambda$ & \#1 (HCG 54b) & $\# 2$ & \#3 (HCG 54a) & $\# 4$ & $\# 5$ & \#6 \\
\hline [OII] & 3727 & $1.65 \pm 0.05$ & $4.72 \pm 0.60$ & $4.54 \pm 0.48$ & $4.53 \pm 0.43$ & $3.86 \pm 0.63$ & $5.04 \pm 0.52$ \\
\hline [NeIII] & 3869 & $0.35 \pm 0.01$ & - & - & - & - & - \\
\hline $\mathrm{H} 8+\mathrm{HeI}$ & 3889 & $0.17 \pm 0.01$ & - & - & - & - & - \\
\hline $\mathrm{H} \epsilon+[\mathrm{NeIII}]$ & 3970 & $0.26 \pm 0.01$ & - & - & - & - & - \\
\hline $\mathrm{H} \delta$ & 4100 & $0.24 \pm 0.02$ & - & - & - & - & - \\
\hline $\mathrm{H} \gamma$ & 4340 & $0.45 \pm 0.01$ & $0.47 \pm 0.04$ & - & - & - & $0.43 \pm 0.04$ \\
\hline [OIII] & 4363 & $0.04 \pm 0.01$ & $0.14 \pm 0.01$ & - & - & - & - \\
\hline $\mathrm{HeI}$ & 4472 & $0.04 \pm 0.01$ & - & - & - & - & - \\
\hline $\mathrm{H} \beta$ & 4861 & $1.00 \pm 0.01$ & $1.00 \pm 0.05$ & $1.00 \pm 0.02$ & $1.00 \pm 0.02$ & $1.00 \pm 0.10$ & $1.00 \pm 0.03$ \\
\hline [OIII] & 4959 & $1.60 \pm 0.02$ & $0.68 \pm 0.06$ & $0.79 \pm 0.04$ & $1.56 \pm 0.07$ & $0.53 \pm 0.09$ & $0.63 \pm 0.04$ \\
\hline [OIII] & 5007 & $4.83 \pm 0.05$ & $2.07 \pm 0.15$ & $1.91 \pm 0.08$ & $3.79 \pm 0.12$ & $1.45 \pm 0.24$ & $2.02 \pm 0.09$ \\
\hline $\mathrm{HeI}$ & 5876 & $0.15 \pm 0.01$ & - & $0.25 \pm 0.03$ & - & - & - \\
\hline$[\mathrm{OI}]$ & 6300 & $0.05 \pm 0.01$ & - & - & - & - & - \\
\hline $\mathrm{H} \alpha$ & 6563 & $2.68 \pm 0.03$ & $3.01 \pm 0.22$ & $2.97 \pm 0.14$ & $2.97 \pm 0.17$ & $2.62 \pm 0.36$ & 2.810 .15 \\
\hline [NII] & 6584 & - & - & $0.36 \pm 0.02$ & - & - & - \\
\hline $\mathrm{HeI}$ & 6678 & $0.03 \pm 0.01$ & $0.26 \pm 0.03$ & - & - & - & - \\
\hline$[\mathrm{SII}]$ & 6717,31 & $0.23 \pm 0.01$ & $0.65 \pm 0.06$ & $1.03 \pm 0.08$ & $1.16 \pm 0.06$ & $0.52 \pm 0.17$ & $0.93 \pm 0.09$ \\
\hline $\mathrm{HeI}$ & 7065 & $0.02 \pm 0.01$ & - & - & - & - & - \\
\hline [AIII] & 7136 & $0.08 \pm 0.01$ & - & - & - & - & - \\
\hline $1.3 * I(6584) / I(3727)$ & & - & 0.07 & 0.10 & - & - & - \\
\hline $\log R_{23}$ & & 0.90 & 0.87 & 0.85 & 0.98 & 0.76 & 0.89 \\
\hline$P=\left[\left(1.3^{*} I(5007) / I(\mathrm{H} \beta)\right) / R_{23}\right]$ & & 0.79 & 0.36 & 0.35 & 0.52 & 0.33 & 0.34 \\
\hline $\log [1.3 * I(5007) / I(3727)]$ & & 0.58 & -0.24 & -0.26 & 0.04 & -0.31 & -0.28 \\
\hline$t[\mathrm{OIII}]\left(10^{4} \mathrm{~K}\right)$ & & 1.05 & - & - & - & - & - \\
\hline$t[\mathrm{OII}]\left(10^{4} \mathrm{~K}\right)$ & & 1.14 & - & - & - & - & - \\
\hline $10^{4} \mathrm{O}^{2+} / \mathrm{H}^{+}$ & & 1.46 & - & - & - & - & - \\
\hline $10^{4} \mathrm{O}^{+} / \mathrm{H}^{+}$ & & 0.36 & - & - & - & - & - \\
\hline $12+\log \mathrm{O} / \mathrm{H}$ & & 8.26 & 8.30 & 8.30 & 8.20 & 8.10:, 8.60: & 8.25: \\
\hline $\operatorname{LogNe^{2+}} / \mathrm{O}^{2+}$ & & -0.67 & - & - & - & - & - \\
\hline
\end{tabular}

${ }^{1}$ Uncertain values are indicated with “:”.

Table 4. Line fluxes and abundances across slit position through HCG 54c-d.

\begin{tabular}{lcccc}
\hline \hline & $\lambda$ & $\# 1$ & $\# 2$ & $\# 3$ \\
\hline$[\mathrm{OII}]$ & 3727 & $3.26 \pm 0.37$ & $4.53 \pm 0.58$ & $3.35 \pm 0.50$ \\
$\mathrm{H} \gamma$ & 4340 & $0.46 \pm 0.04$ & - & - \\
$\mathrm{H} \beta$ & 4861 & $1.00 \pm 0.02$ & $1.00 \pm 0.08$ & $1.00 \pm 0.05$ \\
{$[\mathrm{OIII}]$} & 4959 & $0.76 \pm 0.05$ & $1.64 \pm 0.16$ & $0.80 \pm 0.08$ \\
{$[\mathrm{OIII}]$} & 5007 & $2.57 \pm 0.12$ & $4.04 \pm 0.38$ & $1.98 \pm 0.12$ \\
$\mathrm{H} \alpha$ & 6563 & $3.12 \pm 0.18$ & $2.97 \pm 0.36$ & $2.69 \pm 0.21$ \\
{$[\mathrm{SII}]$} & 6717,31 & $0.78 \pm 0.17$ & $0.91 \pm 0.17$ & $0.79 \pm 0.10$ \\
& & & & \\
$\log R_{23}$ & & 0.82 & 0.99 & 0.77 \\
$P=\left[\left(1.3^{*} I(5007) / I(\mathrm{H} \beta)\right) / R_{23}\right]$ & & 0.51 & 0.54 & 0.43 \\
$\log \left[1.3^{*} I(5007) / I(3727)\right]$ & & 0.01 & 0.07 & -0.12 \\
$12+\log \mathrm{O} / \mathrm{H}$ & & $8.10:, 8.40:$ & 8.20 & $8.10:, 8.45:$ \\
\hline
\end{tabular}

${ }^{1}$ Uncertain values are indicated with ":”.

further test or comparison, since no prediction is given neither for the kinematics nor for the stellar populations/colors of the colliding systems. Here we will try to shed more light in the identification of the involved systems based on the rest of the information provided by our optical and HI data.
We have tried to determine the number of present galaxies using the observed HI mass $\left(\log \left[M(\mathrm{HI})_{\text {obs }} / M_{\odot}\right]=8.75\right)$. We have calculated the expected mass as a function of optical luminosity and morphological type via the relationships obtained by Haynes \& Giovanelli (1984). Assuming that HCG 54 


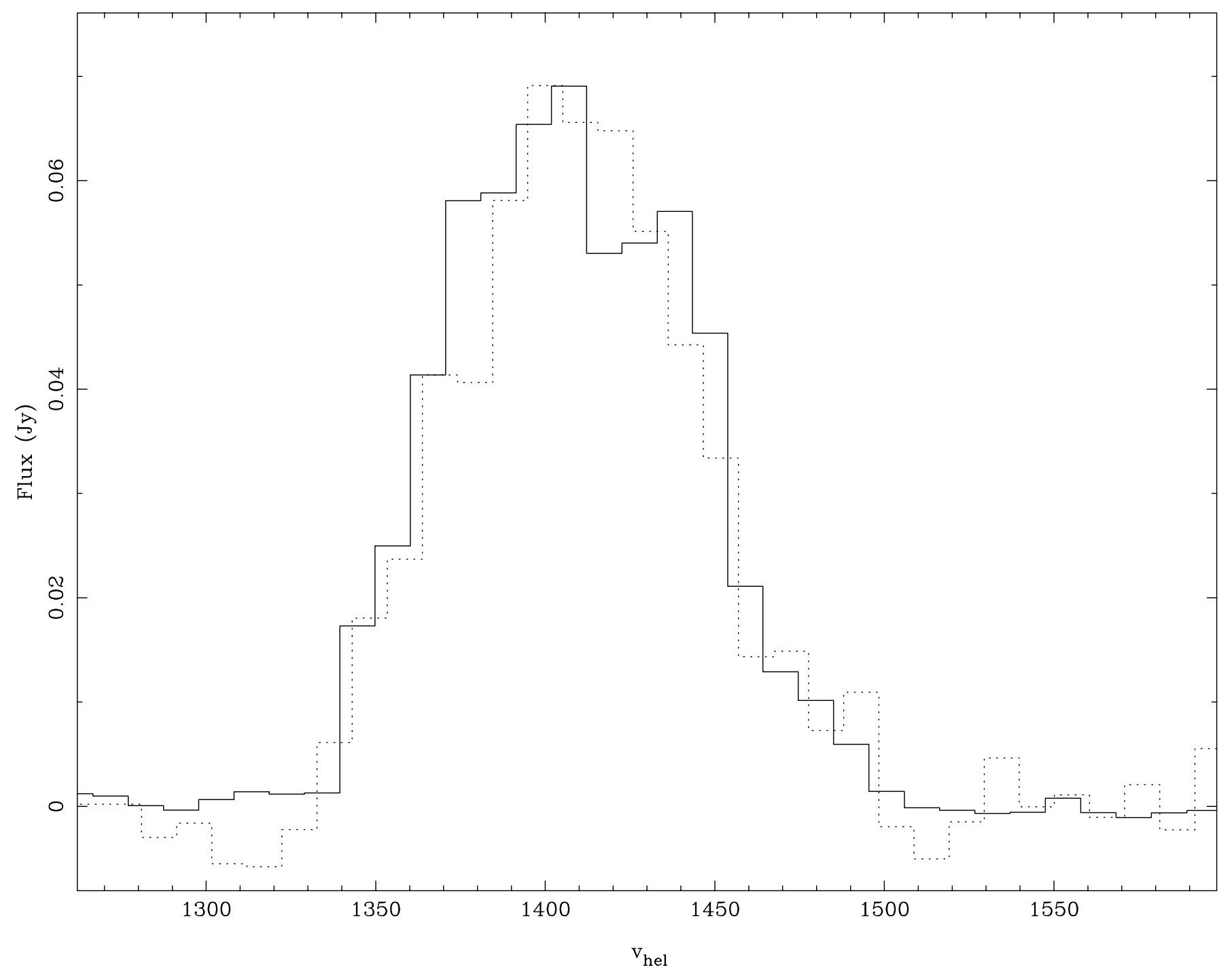

Fig. 10. Integrated $\mathrm{HI}$ emission as a function of the heliocentric velocity for the range where we detect emission. The solid line corresponds to our VLA data, while the dashed ones are single dish data from Huchtmeier (1997).

is composed of 4 irregular galaxies as classified in Hickson catalog, we predict a mass of $\log \left[M(\mathrm{HI})_{\text {pred }} / M_{\odot}\right]=9.16 \pm 0.23$, but for a single irregular galaxy the expected mass would be $\log \left[M(\mathrm{HI})_{\text {pred }} / M_{\odot}\right]=9.19 \pm 0.30$. Unfortunately these cases are indistinguishable since they are within one sigma, and all we can conclude is that the HI content of HCG 54 does not deviate significantly from normal.

HCG 54a is the best defined object of the system, with a defined nucleus, embedded in a very elongated blue component and surrounded by a rounder and redder stellar envelope. This ensemble seems to be aligned with a perturbed but still visible HI velocity gradient. This might indicate that HCG 54a is one of the interacting galaxies, whose disk is a red round component that still keeps memory of the original rotation according to the HI kinematics (Fig. 7b). We find however several arguments against this hypothesis. The atomic gas is decoupled from the stellar component both in morphology and kinematics. The optical emission is displaced to the north by $\sim 1 \mathrm{kpc}$ with respect to the atomic gas, and this shift is too large to be explained only by stripping of the northern $\mathrm{HI}$ into the long tail. Furthermore, the HI velocity gradient does not have a counterpart in the optical velocities. The signature of a rotation curve is not found along any of the observed directions, except for a small velocity gradient of $50 \mathrm{~km} \mathrm{~s}^{-1}$ in the inner 5" of HCG 54a (see Sect. 3.2). The HI radial velocities along the direction of the optical spectra differ from the optical ones by $\sim 30 \mathrm{~km} \mathrm{~s}^{-1}$, also consistent with a decoupling between the stellar and gaseous components. Finally, since the HI velocity gradient is the only trace of a possible disk system in the central part of HCG 54, then the second disk would be a minor body when compared with HCG 54a, in contradiction with the equal masses involved in the simulations. Furthermore, if the observed HI velocity gradient of $\sim 180 \mathrm{~km} \mathrm{~s}^{-1}$ had to be attributed to a single galaxy, it would be more characteristic of an Sb type system, which is not observed here.

A more plausible scenario is that we are observing a mix of the two components of the original systems, that have almost coalesced and now present a similar distribution as found in the simulations. The irregular shape of the optical velocity curve supports an advanced merger stage. HCG 54b shows a 

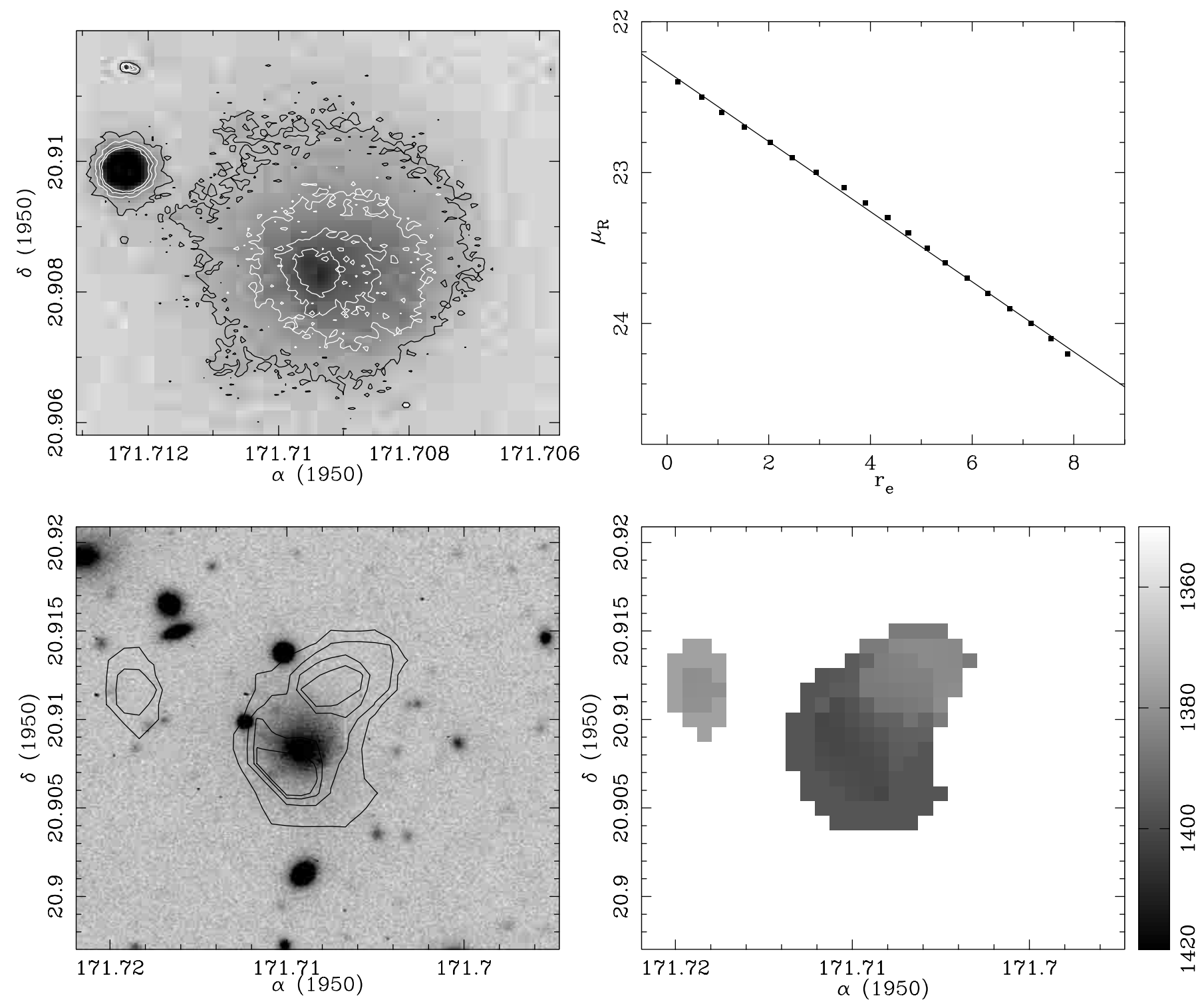

Fig. 11. a) Upper-left: $R$ band emission of A1127+2054 in greyscale with overlapped isophotes 22.6, 22.9, 23.3, 24.1 mag arcsec $^{-2}$. b) Upper right: light profile of this galaxy in the $R$ band as a function of the equivalent radius. c) Lower left: integrated HI emission of this galaxy where the contours correspond to 3.3, 6.6, 10.0 and $11.6 \times 10^{20}$ atoms $\mathrm{cm}^{-2}$, and overlapped on the $R$ image. d) Lower-right: map of the first-order moment of the HI radial velocity field. The scale goes as in the wedge where the numbers indicate heliocentric velocities in $\mathrm{km} \mathrm{s}^{-1}$.

clear WR feature in the spectrum, indicative of a recent and strong burst of star formation that was possibly triggered by the interaction, while the underlying population, if present, is negligible, as suggested by the very high equivalent widths of the brightest emission lines (see Sect. 3.1.). In fact we found a possible signature of gas infall in our spectra (see Sect. 3.2) that would be feeding the recent violent star formation observed. HCG 54c and d do not have a well defined photometric structure, but consist of an aggregate of knots, and have HII regions features in their spectra. The very blue color of HCG 54d suggests a pure star formation episode younger than several million years old. They plausibly constitute the debris of the interacting systems. The HI component of the kinematics we observe might be the relic of the orbital motions of the interacting systems. However projected radial motions cannot be negelected and detailed numerical simulations including a kinematical study are needed to interpret the data.

In summary, no clear individual galaxies could be identified toward HCG 54 while strong signs of interactions and a merger are found. The overall morphology is in good agreement with the model by Hernquist \& Spergel (1992), and we propose that HCG 54 is in the final stages of a merger of two similar mass disk systems.

\subsection{Formation of the HI tidal tail}

While a chance projection is possible, the location of A1127+2054, a dwarf galaxy at the redshift of the group, closely aligned with the large $\mathrm{HI}$ tidal tail is strongly suggestive of its role in shaping the appearance and the extent of the HI 
tail. A tidal tail pointing back at the responsible intruder is a commonly observed generic feature, particularly soon after the closest approach, as the exchange of momentum between the intruder and the tidally disrupted material tends to bring them close to the common area in the phase space.

A $1127+2054$ is not likely to be a tidal dwarf since it is not located within the tail and has its own rotating HI disk or cloud. The fact that the associated HI feature is not well centered on the stellar body is consistent with the tidal disruption scenario. No stellar counterpart to the HI tail is detected down to a surface brightness level of $27 \mathrm{mag} /\left({ }^{\prime \prime}\right)^{2}$ in $R$ along its length.

This tidal disruption scenario has some potential difficulties however. Assuming an inclination of $30^{\circ}$, the estimated dynamical mass for A $1127+2054$ inside $2.1 \mathrm{kpc}$ radius is only about $4 \times 10^{8} M_{\odot}$, which is about $4 \%$ of the dynamical mass of HCG 54. On the other hand, the estimated HI mass alone for the $20 \mathrm{kpc}$ long tidal tail is about $10^{8} M_{\odot}$ (see Sect. 3.3), and raising such a massive tidal tail from the gravitational potential of HCG 54 may require a far more massive perturber.

An alternative scenario for the formation of the large HI tail is the merger of two equal mass progenitors as we have already proposed in the previous section in order to account for the disrupted stellar features around HCG 54. In numerical simulations of galaxy mergers including gas particles such as by Mihos \& Hernquist (1996), formation of one or more massive gaseous tails is commonly seen. For example, the projected appearance of the HI tail with respect to the stellar remnant in HCG 54 is similar to the " $t \sim 70$ " stages shown in Figs. 1 and 2 of Mihos \& Hernquist (when mirrored about the vertical axis). This corresponds to a late stage in this particular simulation where the stellar cores finally merge, similar to what we infer in HCG 54. If we are allowed to press this comparison a little further, the extended HI structures seen to the south and southeast of the stellar body may be interpreted as the tidal tail emerging from the second progenitor, as seen in the simulation. Of course, these inferences are qualitative at best since the initial conditions of the Mihos \& Hernquist simulation are likely to be different from those of the merger involved in HCG 54.

In this scenario, an active role of A $1127+2054$ in shaping the appearance of the large HI tail is still allowed. For example, a dwarf companion UGC 957 is found within one of the large HI tails resulting from the merger of two massive disk galaxies in NGC 520 (see Hibbard \& van Gorkom 1996) even though UGC 957 may be only a third party to the ongoing or recent merger.

\section{Conclusions}

We propose that HCG 54 is a system that consists of at least two late type galaxies plus a dwarf companion involved in recent tidal interactions and a merger. Two low mass disk systems merged and induced the formation of a central blue, bar-like structure embedded in a red, round stellar envelope, outside which lies several stellar shells. A luminous starburst is associated with HCG 54b while HCG 54c and d are the remnant debris of the recent merger undergoing modest star formation activity. This merger is probably in a very advanced state. The $20 \mathrm{kpc}$ long gaseous tidal tail discovered in HI may be a result of the same merger or driven by the dwarf galaxy A1127+2054 found at a projected distance of $8 \mathrm{kpc}$ from the tip. In either scenario, it is likely playing a role in shaping the appearance of this tidal tail. Unfortunately current theoretical or numerical models do not provide information on the kinematics or the distribution of induced star formation in low mass disk mergers. Few similar systems have been discussed in the literature, and the present data should provide valuable inputs for future modeling of mergers involving low mass galaxies.

Acknowledgements. We wish to thank Dr. Masegosa and Dr. Márquez for the help with the R observations at the NOT and their valuable comments. LV-M, AO and JP are partially supported by DGI (Spain) Grant AYA2000-1564 and Junta de Andalucía (Spain). JMV is partially supported by DGI (Spain) Grant AYA2001-3939-C03-01 and Junta de Andalucía (Spain).

\section{References}

Arkhipova, V. P., Afanasev, V. L., Dostal, V. A., et al. 1981, Sov. Astron., 25, 277

Charmandaris, V., \& Appleton, P. N. 1998, ApJ, 460, 686

Fukugita, M., Shimasaku, K., \& Ichikawa, T. 1995, PASP, 107, 945

Haynes, M. P., \& Giovanelli, R. 1984, AJ, 89, 758

Hernquist, L., \& Quinn, P. J. 1987, ApJ, 312, 17

Hernquist, L., \& Spergel, D. N. 1992, ApJ, 399, L117

Hibbard, J. E., \& van Gorkom, J. H. 1996, AJ, 111, 655

Hickson, P. 1982, ApJ, 255, 382

Hickson, P., Kindl, E., \& Auman, J. R. 1989, ApJS, 70, 687

Hickson, P., Mendes de Oliveira, C., Huchra, J. P., \& Palumbo, G. 1992, ApJ, 399, 353

Higdon, J. L. 1996, ApJ, 467, 241

Homeier, N. L., \& Gallagher, J. S. 1999, ApJ, 522, 199

Huchtmeier, W. K. 1997, A\&A, 325, 473

Kemp, S. N., \& Meaburn, J. 1993, A\&A, 274, 19

Kobulnicky, H. A., Kennicutt, R. C., \& Pizagno, J. L. 1999, ApJ, 514, 544

Mihos, J. C., \& Hernquist, L. 1996, ApJ, 464, 641

Mulder, \& van Driel 1996, A\&A, 309, 403

Pagel, B. E. J., Edmunds, M. G., \& Smith, G. 1980, MNRAS, 193, 219

Pilyugin, L. S. 2000, A\&A, 362, 325

Plana, H., Amram, P., Mendes de Oliveira, C., \& Balkowski, C. 2000, AJ, 120, 621

Quinn, P. J. 1984, ApJ, 279, 596

Schaerer, D., \& Vacca, W. D. 1998, ApJ, 497, 618

Schweizer, F. S., \& Seitzer, P. 1988, ApJ, 328, 88

Tonry, J., \& Davies, M. 1979, AJ, 84, 1511

Verdes-Montenegro, L., Yun, M. S., Perea, J., del Olmo, A., \& Ho, P. T. P. 1998, ApJ 497, 89

Verdes-Montenegro, L., Yun, M. S., Williams, B. A., et al. 2001, A\&A, 377, 812

Vílchez, J. M., \& Iglesias-Páramo, J. 1998, ApJS, 117, 1

Williams, B. A., \& Van Gorkom, J. 1988, AJ, 95, 352

Yun, M. S., Reddy, N. A., \& Condon, J. J. 2001, ApJ, 554, 803 